ARTICLE

\title{
A TALE/HOX code unlocks WNT signalling response towards paraxial mesoderm
}

\author{
Luca Mariani ${ }^{1,3}$, Xiaogang Guo ${ }^{1,3}$, Niels Alvaro Menezes ${ }^{1}$, Anna Maria Drozd (1) ${ }^{1}$, Selgin Deniz C,akal (1) ${ }^{1}$,
} Qinhu Wang (iD ${ }^{2} \&$ Elisabetta Ferretti ${ }^{1 凶}$

One fundamental yet unresolved question in biology remains how cells interpret the same signalling cues in a context-dependent manner resulting in lineage specification. A key step for decoding signalling cues is the establishment of a permissive chromatin environment at lineage-specific genes triggering transcriptional responses to inductive signals. For instance, bipotent neuromesodermal progenitors (NMPs) are equipped with a WNT-decoding module, which relies on TCFs/LEF activity to sustain both NMP expansion and paraxial mesoderm differentiation. However, how WNT signalling activates lineage specific genes in a temporal manner remains unclear. Here, we demonstrate that paraxial mesoderm induction relies on the TALE/HOX combinatorial activity that simultaneously represses NMP genes and activates the differentiation program. We identify the BRACHYURY-TALE/HOX code that destabilizes the nucleosomes at WNT-responsive regions and establishes the permissive chromatin landscape for de novo recruitment of the WNT-effector LEF1, unlocking the WNTmediated transcriptional program that drives NMPs towards the paraxial mesodermal fate.

\footnotetext{
${ }^{1}$ Novo Nordisk Foundation Center for Stem Cell Biology (DanStem), University of Copenhagen, Copenhagen, Denmark. ${ }^{2}$ State Key Laboratory of Crop Stress Biology for Arid Areas and College of Plant Protection, Northwest A\&F University, Yangling, Shaanxi, China. ${ }^{3}$ These authors contributed equally: Luca Mariani, Xiaogang Guo. ${ }^{凶}$ email: elisabetta.ferretti@sund.ku.dk
} 
n mammals, skeletal muscles and vertebrae originate from the paraxial mesoderm, which includes unsegmented pre-somitic mesoderm (PSM) and somites ${ }^{1-3}$. PSM formation, which represents the earliest event of musculoskeletal development, relies on the availability of expanding neuromesodermal progenitors (NMPs) that also contribute to the spinal $\operatorname{cord}^{3-6}$. NMPs reside in the caudal lateral epiblast (CLE) and tailbud, from where they sustain axial elongation $4,7,8$. Intricate molecular circuits regulated by WNT signalling and involving the transcription factors (TFs) HOX, CDX, SOX2, BRACHYURY (T-BRA), TBX6 and MSGN1, balance NMP expansion and differentiation ${ }^{9-17}$. NMPs can adopt neural fate by downregulating T-Bra and maintaining Sox2 expression. Conversely, NMPs acquiring PSM fate retain T-Bra expression, downregulate Sox2 and upregulate Tbx6 and Msgn1, transitioning into nascent mesodermal progenitor cells (MPCs) that fuel PSM development ${ }^{14,18}$. Ultimately, somite formation depends on the speed by which NMPs leave the CLE to acquire paraxial mesoderm fate ${ }^{1}$.

HOX TFs have been historically implicated in axial elongation, with their sequential activation playing a fundamental role in timing PSM development ${ }^{16,19,20}$. Posterior Hox genes (Hox10-13) reduce mesoderm ingression by repressing WNT and are responsible for body axis termination ${ }^{11,21}$. Conversely, the anterior Hox genes (Hox1-8) control paraxial mesoderm formation by modulating cell ingression into the primitive streak $(\mathrm{PS})^{19}$. Nevertheless, the mechanism by which Hox genes promote PSM specification remains largely unknown. TFs belonging to the TALE (three-amino acid loop extension) family like the PBX and MEIS factors have distinct ability to interact with HOX proteins and provide HOX with DNA-binding specificity $^{22}$. Given that PBX proteins are obligate anterior HOX cofactors, they represent prominent candidates for controlling the distinct response to individual HOX factors ${ }^{23}$. PBX1, PBX2 and PBX3 play fundamental roles during organogenesis $22,24,25$. Compound deletion of $\mathrm{PBX} 1$ and $\mathrm{PBX} 2$ (hereafter PBX) uncovers a crucial role for these factors in axial/appendicular patterning 24,25 , a surprisingly late phenotype as they are widely expressed at earlier embryonic stages ${ }^{26}$. Importantly, their role in mesodermal lineage specification remains elusive. Given the broad expression of PBX, it is still unclear how cooperative interactions with HOX provide lineage specificity.

By employing in vitro differentiation systems and in vivo mouse models, we show that the patterning abnormalities in $P b x 1 / 2$ double mutant embryos (hereafter $P b x$ ) can be traced back to the PSM specification stage. In Pbx mutants, NMPs exhibit reduced ability to generate paraxial mesoderm, being unable to migrate, remaining trapped in the tailbud in a NMP/ MPC transition state. Using single-cell sequencing, chromatin accessibility analyses and iterative rounds of DNA:protein interaction assays, we demonstrate that TALE/HOX proteins generate the DNA-binding context for the recruitment of the WNTeffector LEF1, and consequently promote the expression of PSM genes, including the master regulator Mesogenin1 $(M \operatorname{sgn} 1)^{10,15}$. Ultimately, the activation of the paraxial mesoderm gene regulatory networks (GRNs) rely on the activity of a WNT-HOX integrated code, which drives a multistep process involving changes in the chromatin landscape at the WNT-responsive elements supporting the shift from NMP expansion to paraxial mesoderm differentiation. Thus, TALE/HOX act as a molecular switch that triggers alternative WNT signalling cellular responses, unlocking PSM genes and promoting somite formation.

\section{Results}

PBX proteins control the formation of the nascent paraxial mesoderm. Addressing how Hox genes influence WNT signalling is critical for paraxial mesoderm formation and somitogenesis ${ }^{11,19}$. The
HOX cofactors PBX are broadly expressed in the tailbud of embryonic day (E) 8.5 mouse embryos, including PS and NMPs (Supplementary Fig. 1a), questioning their function in the early stages of somitogenesis. To dissect the role of PBX at early stages, we inactivated $P b x 1$ in $P b x 2$ mutant mice using the Sox ${ }^{C r e}$ allele to obtain $P b x 1^{-/-} ; P b x 2^{-/-}$double-knockout $(P b x-D K O)$ and $P b x 1^{-/}$ ${ }^{-} ; P b x 2^{+/-}$compound mutant ( $P b x$-com) embryos. $P b x$-DKO display shortened trunk, enlarged tailbud and rudimentary somites (Fig. 1a and Supplementary Fig. 1b). Furthermore, FOXC2 and UNCX4.1 immunofluorescence (IF) revealed abnormal posterior somite morphogenesis in E8.5 Pbx-DKO and Pbx-com (Fig. $1 \mathrm{~b}$ and Supplementary Fig. 1c). To pinpoint the role of PBX proteins in PSM development (Fig. 1c), we performed single-cell RNA sequencing (scRNA-seq) on cells isolated from the tailbuds of control and $\mathrm{Pbx}$ mutants at E8.5 and E9.0 (Fig. 1d and Supplementary Data 1). After stringent quality filtering, batch-effect correction and data integration of all sequenced embryos, unsupervised clustering analyses revealed 13 different populations, including NMPs, MPCs and PSM (Fig. 1e, Supplementary Fig. 1d, e and Supplementary Data 2). Each embryo contributed to every cluster, irrespective of the genotype. However, controls and mutants exhibited markedly different proportions of cell types populating each cluster. Specifically, $P b x-D K O$ and $P b x$-com mutant cells were overrepresented in the posterior PSM cluster, while being largely reduced in anterior PSM, suggesting a block on PSM maturation. Quantification of cluster composition confirmed that cells displaying MPC features (T-Brapos; $\left.T b x 6^{p o s}\right)$ significantly accumulate in the posterior PSM cluster of $P b x-D K O$ and $P b x$-com at the expense of anterior PSM (Fig. 1f). Specifically focussing on NMPs, MPCs and PSM cell populations revealed that the $P b x-D K O$ and $P b x$-com NMPs and MPCs/pPSM clusters included cells that exhibited a mixed NMP/MPC profile, expressing high levels of T-Bra, Sox2, Wnt $3 a, T b x 6$ and $5^{\prime}$ Hox genes (Fig. $1 \mathrm{~g}$ and Supplementary Fig. 1f-h). To understand the transition of NMPs towards PSM, we applied pseudotemporal ordering analyses and confirmed defective paraxial mesoderm differentiation in $P b x-D K O$, resulting in inflation of the progenitor reservoir at the expense of mature PSM (Fig. 1h and Supplementary Fig. 1i).

The impairment of PSM formation and the increased number of MPCs with NMP features in $P b x-D K O$ and $P b x$-com prompted us to address whether the spatial organisation of the progenitor zone was affected ${ }^{27}$. By IF, we observed an expansion of T-BRA ${ }^{\text {pos;SOX }} 2^{\text {pos }}$ NMPs in the $P b x$-DKO and $P b x$-com tailbuds (Fig. 2a and Supplementary Fig. 2a). By contrast, the mesodermal marker TBX6 was strongly downregulated in the PSM and upregulated in the CLE of $P b x-D K O$ and $P b x$-com mutants (Fig. 2a), confirming the accumulation of aberrant progenitors observed by scRNA-seq. The distribution of progenitor populations assessed in the caudal and medial parts of the CLE, where cells preferentially adopt a PSM fate, revealed that in $P b x-D K O$ and Pbx-com the number of NMPs (T-BRApos;SOX2pos) and MPCs (T-BRApos;TBX6 ${ }^{\text {pos }}$ ) increased, while the amount of mature PSM (TBX6 ${ }^{\text {pos}}$ ) cells was dramatically reduced (Fig. 2b, c and Supplementary Fig. 2b). Notably, the proportion of T-BRApos;TBX6 ${ }^{\text {pos }}$ cells relative to TBX6 ${ }^{\text {pos }}$ cells was significantly enhanced in $P b x-D K O$ and $P b x$-com, suggesting an impaired maturation of MPCs (Fig. 2c). Furthermore, NMPs robustly accumulate in the mutant posterior tailbuds, as opposed to the fewer NMPs observed in controls (Fig. 2d). Thus, the analysis of the progenitor allocation in $P b x$ mutant embryos corroborated the scRNA-seq findings (Fig. 1e-h), and provided evidence that $P b x$ $D K O$ and $P b x$-com NMPs are hindered in their progress towards PSM, remaining trapped in the CLE in a NMP/MPC state.

Given that WNT3A supports both NMP maintenance and PSM differentiation $^{14,18}$ (Supplementary Fig. 2c), we tested whether the PSM defects in the $\mathrm{Pbx}$ mutants were the consequence of perturbed 
a

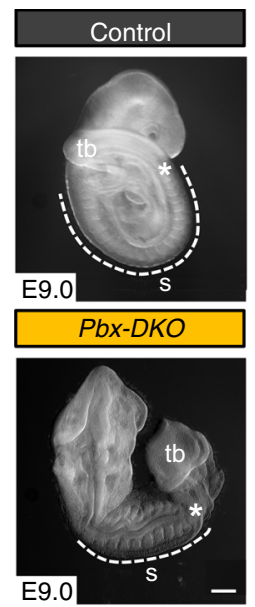

b

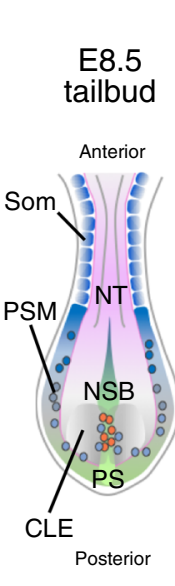

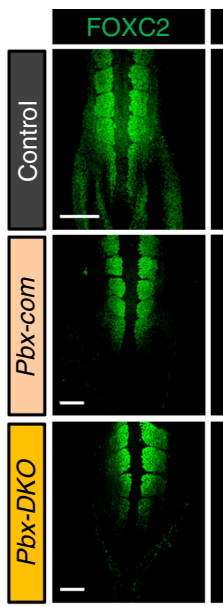

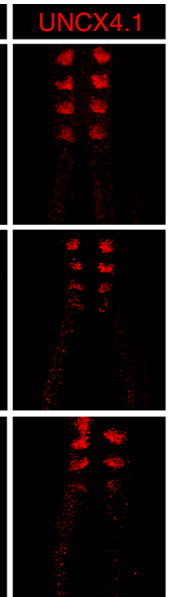

C

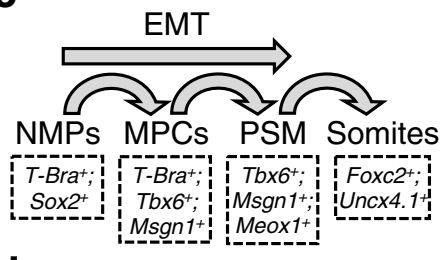

d

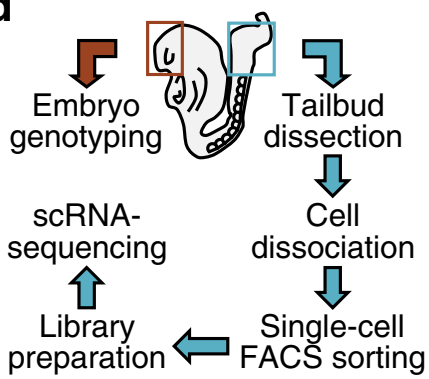

e

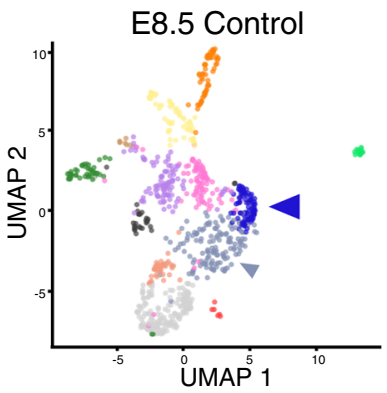

E8.5 Pbx-com

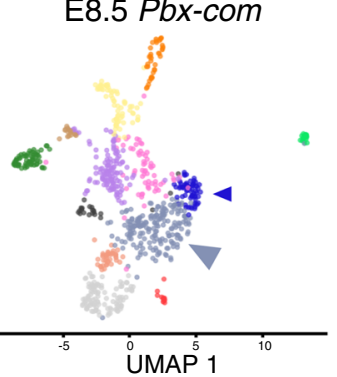

E9.0 Control

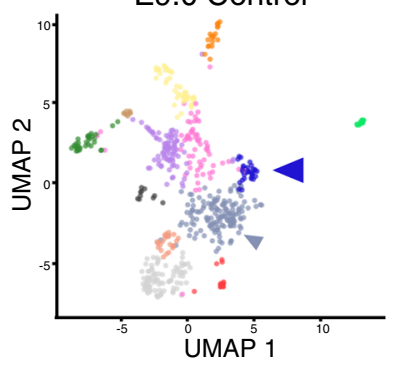

E9.0 Pbx-DKO
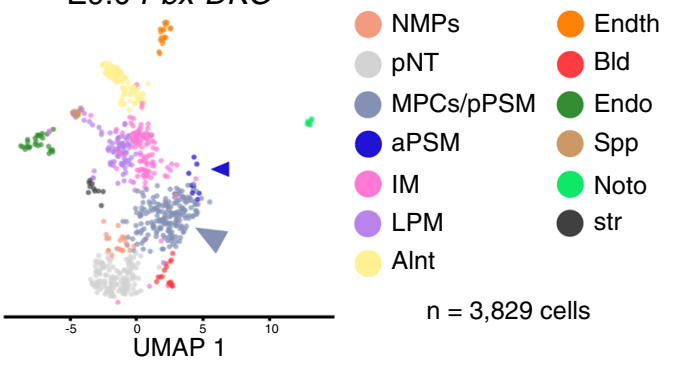

h

g

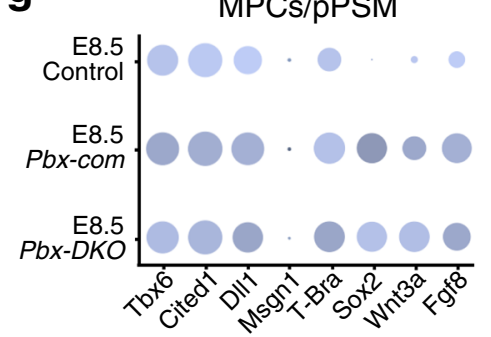

\% of cells expressing 255075 marker genes

Average expression
- 0

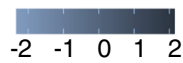

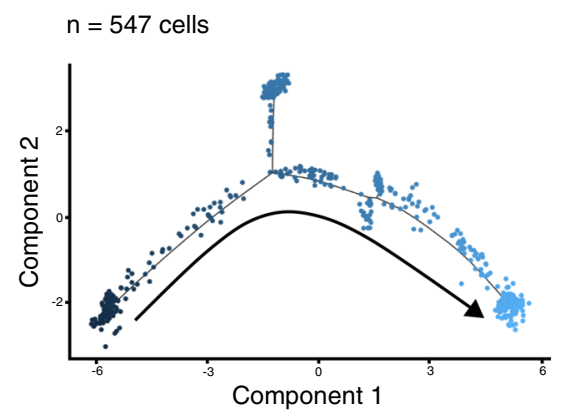

Pseudotime

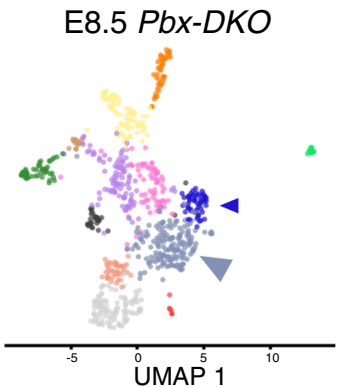

MPCs

E8.5 E9.0
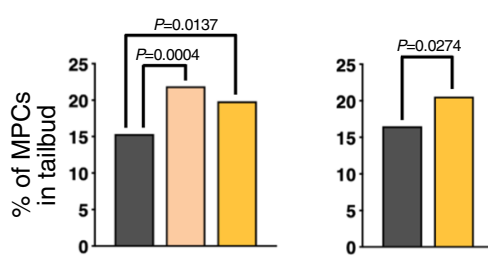

aPSM

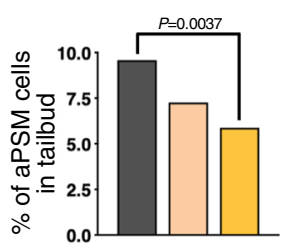

Control

Pbx-com

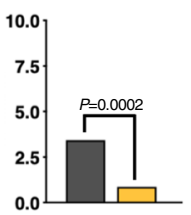

$P b x-D K O$

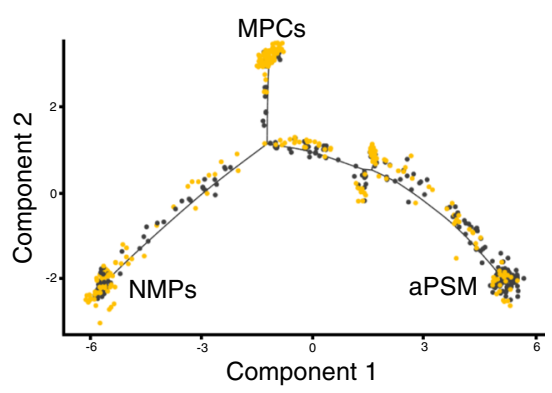

Control

$P b x-D K O$

WNT expression, and found that Wnt $3 a$ expression domain was increased in the $P b x$-com tailbud (Supplementary Fig. 2d), in agreement with the observed progenitor zone expansion (Fig. 2a, d). Therefore, although WNT signalling is a major mediator of PSM specification, the phenotypes of $P b x$ mutants are not associated with the depletion of the Wnt $3 a$ ligand, suggesting that PBX could have a role in modulating how NMPs respond to this pathway. Remarkably, $P b x$ mutants have an expanded NMP population, implicating PBX in the regulation of the WNT-dependent NMP-toPSM transition. 
Fig. 1 PBX proteins control the transition of NMPs towards PSM, underlying the acquisition of paraxial mesoderm identity. a Gross morphology of E9.0 $P b \times 1^{-/-} ; P b \times 2^{-/-}(P b x-D K O)$ embryos showing enlarged tailbud (tb), shortened trunk and rudimentary somites (s). Scale bar: $200 \mu \mathrm{m}$. $\mathbf{b}$ Dorsal views of E8.5 $\mathrm{Pbx} 1^{-/-} ; \mathrm{Pbx} 2^{+/-}(\mathrm{Pbx}-\mathrm{Com})$ and $\mathrm{Pbx}$-DKO mouse embryos stained with FOXC2 (green) and UNCX4.1 (red) antibodies display abnormal

somitogenesis. The cartoon on the left is a schematic representation of the E8.5 mouse tailbud. CLE caudal lateral epiblast, NSB node-streak border, PS primitive streak, PSM pre-somitic mesoderm, Som somites, NT neural tube, A anterior, P posterior. Scale bars: $70 \mu \mathrm{m}$. In $\mathbf{a}$ and $\mathbf{b}$, asterisks denote the newly generated somites. c Schematics illustrating paraxial mesoderm differentiation from bipotent NMPs to somites, showing intermediate states and associated lineage markers. EMT epithelial-to-mesenchymal transition. $\mathbf{d}$ Strategy used to perform single-cell RNA-seq (scRNA-seq) of mouse embryonic tailbuds. e ScRNA-seq of E8.5/E9.0 control, Pbx-com and Pbx-DKO embryonic tailbuds. Unsupervised clustering and UMAP visualisation reveal reduced aPSM (dark blue arrowhead) and expanded MPCs/pPSM (light blue arrowhead) cell populations in Pbx mutants. Individual cells are coloured according to annotated cluster identities: NMPs neuromesodermal progenitors, pNT pre-neural tube, MPCs/pPSM mesodermal progenitor cells/posterior pre-somitic mesoderm, aPSM anterior pre-somitic mesoderm, IM intermediate mesoderm, LPM lateral plate mesoderm, Alnt allantois, Endth endothelial cells, Bld blood, Endo endoderm, Spp splanchnopleura, Noto notochord, str stressed cells. f Percentage of MPCs and aPSM cells in control (black), Pbx-com (light pink) and Pbx-DKO (yellow) tailbuds at E8.5 and E9.0. P-values indicated in the figure were calculated by two-sided Fisher's exact test. $\mathbf{g}$ Expression analysis of selected lineage markers within the MPCs/pPSM cluster isolated from control and Pbx mutant tailbuds. Dot plot shows the relative expression of the NMP markers T-Bra, Sox2, Wnt3a, Fgf8, and the mesodermal genes Tbx6, Cited1, DIl1, Msgn1, indicating co-expression of NMP and MPC markers in $\mathrm{Pbx}$-com and $\mathrm{Pbx}-\mathrm{DKO}$ embryos. Colour bar indicates the intensity associated with normalised expression values. $\mathbf{h}$ Monocle's pseudotemporal ordering of paraxial mesoderm-related clusters displaying the progressive maturation of control cells (black) from NMPs to aPSM and the defective NMP-to-PSM transition of $P b x-D K O$ cells (yellow).

PBX proteins promote the transition from NMPs towards PSM. To address PBX function during PSM differentiation, we took advantage of our recently developed in vitro differentiation protocol (Mariani, L. et al., in preparation ${ }^{28}$ ) that efficiently drives mouse epiblast stem cells (EpiSCs) towards PSM (Fig. 3a and Supplementary Fig. 3a). This system allowed us to capture, with high temporal resolution, the transition from NMPs to nascent PSM that would be otherwise difficult to pinpoint in vivo due to the limited number of progenitors. ScRNA-seq analyses at different time-points identified NMPs by the co-expression of T-Bra, Sox2, Wnt3a, Nkx1-2 and Cdx2 at $24 \mathrm{~h}$ (Fig. 3b, c and Supplementary Data 3). These NMPs expressed Cdx1, Cdh1, Fst and the anterior Hox genes (Hoxal-b1), a signature characteristic of E8.5 NMPs ${ }^{18}$. At 36 and $48 \mathrm{~h}$, NMPs expressing posterior Hox genes (Hox6-9) compatible with a E9.5 NMP or pre-neural signature coexist with cells transitioning towards PSM $^{18}$, marked by Tbx6, Foxc2 and Meox1 (Fig. 3b, c). To confirm that our in vitro model replicated genuine in vivo PSM differentiation, we assessed the capacity of these cells to generate somite-derived tissues, by differentiating PSM towards sclerotome and dermomyotome, which give rise to axial skeleton and muscles respectively (Supplementary Fig. 3b). Populations of sclerotome-like cells expressing Foxc2, Pax1 and Pax9, and dermomyotome-like cells marked by Pax3 and Pax7 were readily detectable following 5 days of differentiation, confirming the potency of the progenitors (Supplementary Fig. 3c, d).

To address PBX role in PSM formation, we established $P b x$ $D K O$ cell lines, either by inactivating $P b x 1$ and $P b x 2$ using CRISPR/Cas9 (Fig. 3d) or by CRE-mediated recombination of $P b x 1^{f l o x} / f l o x ; P b x 2^{-/-}$derived EpiSCs (Supplementary Fig. 3e). Real-time quantitative PCR (RT-qPCR) and IF revealed impaired ability of $P b x-D K O$ cells to generate dermomyotome and sclerotome, mimicking the $P b x$-DKO and $P b x$-com embryonic defects (Supplementary Fig. 3f-h) ${ }^{24,25}$.

We used scRNA-seq analyses along PSM differentiation to test if $P b x-D K O$ phenotypes are recapitulated in vitro (Fig. 3e), focussing on the transition from NMPs to PSM. Temporal trajectory reconstruction along differentiation showed that wildtype (WT) NMPs efficiently differentiate towards PSM, whereas $P b x-D K O$ cells fail to reach the PSM state and accumulate as progenitors co-expressing $\mathrm{T}-\mathrm{Bra}$ and Sox2 (Fig. 3f, g). IF staining and quantification confirmed that PBX loss strongly impairs PSM differentiation, resulting in a higher proportion of NMPs and MPCs at the expense of PSM (Fig. 3h, i), recapitulating the phenotypes observed in vivo and highlighting the critical role of PBX proteins in specifying PSM.
PBX proteins are dynamically recruited to PSM genes. To identify PBX targets during PSM differentiation, we performed chromatin immunoprecipitation followed by deep sequencing (ChIP-seq) using PBX1 and PBX2 antibodies at different timepoints (Fig. 4a). The number of PBX1 peaks increased during differentiation (Supplementary Fig. 4a), with a maximum number of binding events occurring at $24 \mathrm{~h}$ : the stage when mesodermal fate is established. The functional readout of PBXbinding events was assessed by RNA-sequencing (RNA-seq) of WT and $P b x-D K O$ cells at 12 and $24 \mathrm{~h}$ (Fig. $4 \mathrm{a}-\mathrm{c}$ and Supplementary Fig. 4 b, c). To pinpoint the genes directly modulated by PBX, we integrated the ChIP-seq and RNA-seq datasets and identified 251 differentially regulated targets (Supplementary Data 4). GO-term analyses of the downregulated genes confirmed PBX requirement in PSM differentiation, somite development and segmentation (Fig. $4 \mathrm{~b}$ and Supplementary Fig. $4 \mathrm{~b}$ ). By contrast, loss of PBX resulted in increased expression of neuronal and NMP genes like Sox 2 and $C d x 2$, suggesting that PBX may be important for balancing the alternative fates of NMPs (Fig. 4c and Supplementary Fig. 4c). As previous reports have suggested that PBX proteins possess pioneer factor activity ${ }^{29-31}$, we assessed the PBX-dependent changes in chromatin accessibility at the PBX-bound sites. We performed Assay for Transposase-Accessible Chromatin using sequencing (ATAC-seq) at different time-points (Fig. 4d) and compared chromatin accessibility of the PBX target genes misregulated in $P b x-D K O$ cells (Fig. 4e, f). Regulatory regions associated with upregulated genes in $P b x-D K O$ were open in EpiSCs and remained accessible in differentiated WT and $P b x-D K O$ cells (Fig. 4f). By contrast, loci associated with downregulated genes in $P b x-D K O$ were closed in EpiSCs and became increasingly accessible during PSM differentiation peaking at $24 \mathrm{~h}$ in WT, but not in Pbx-DKO cells (Fig. 4e). Thus, by enabling chromatin opening at PSM loci, PBX proteins could prime regulatory regions for the recruitment of lineage-specific TFs. These results substantiate the previously suggested role of PBX as pioneer factors ${ }^{29-31}$, but raise the question of how these broadly expressed proteins act at specific loci. As canonical transcriptional regulation is known to be driven by cooperativity, we searched for factors that could cooperate with PBX to promote chromatin accessibility at key PSM regulatory regions ${ }^{32}$. De novo motif analyses of the PBX-bound regions revealed dynamic changes in the TF-binding site enrichment during differentiation, suggesting cooperative binding with distinct partners at different time-points (Fig. $4 \mathrm{~g}$ and Supplementary 
a
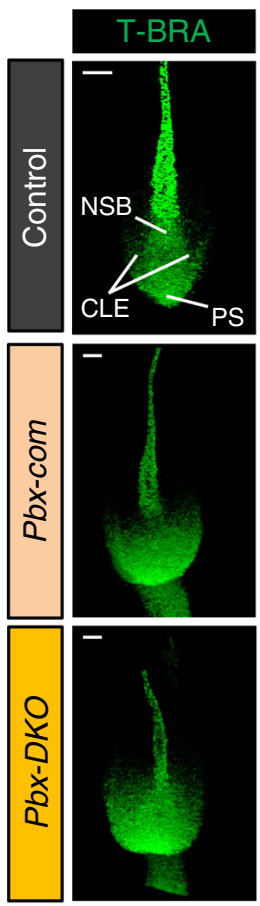

b

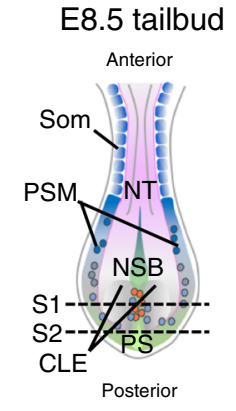

\section{Cell Molecular}

NMPs T-BRA + SOX2 MPCs T-BRA $+/ T B X 6$ PSM TBX6+
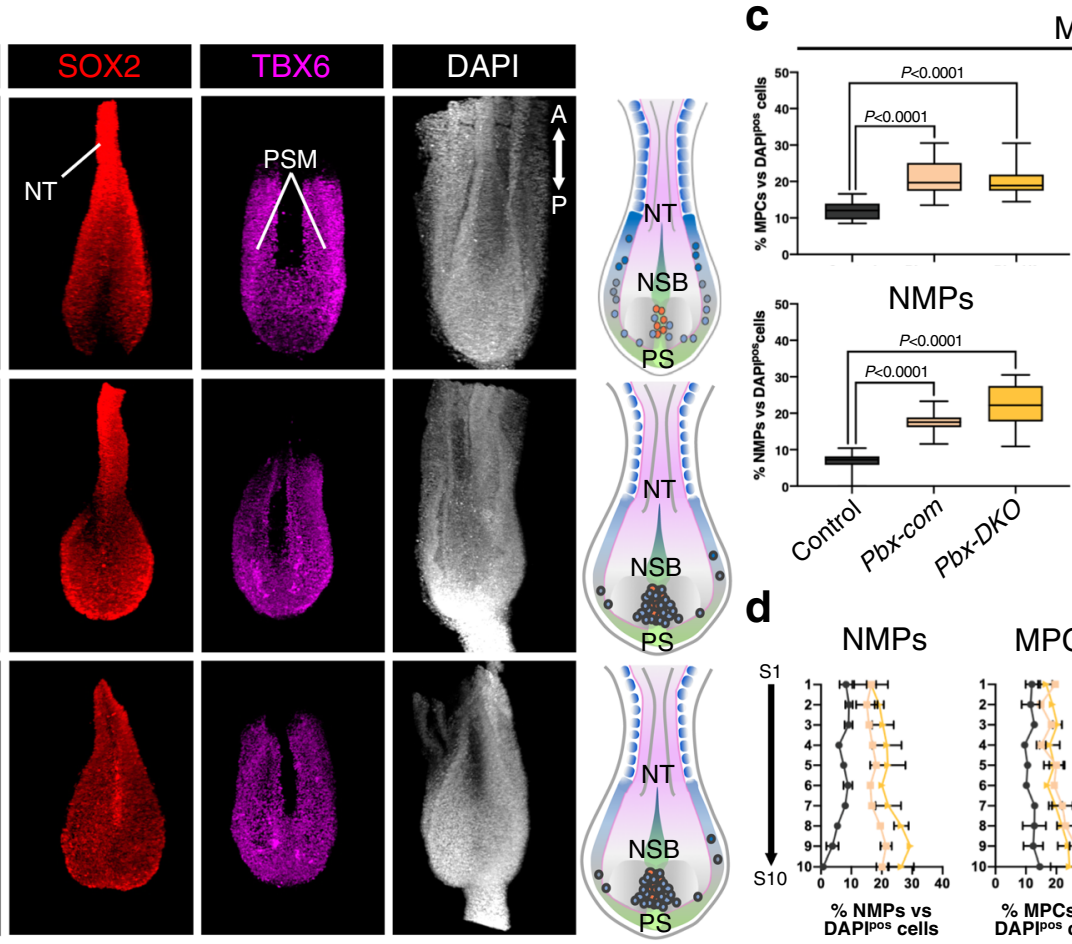

MPCs
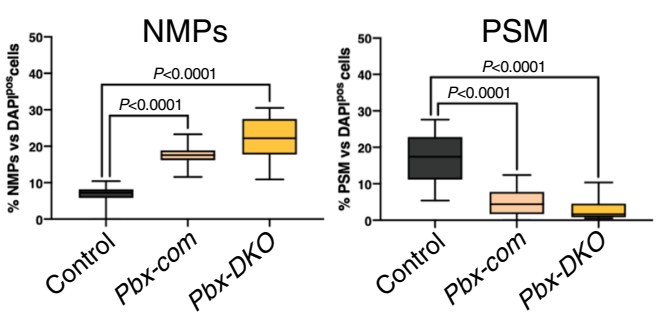

d
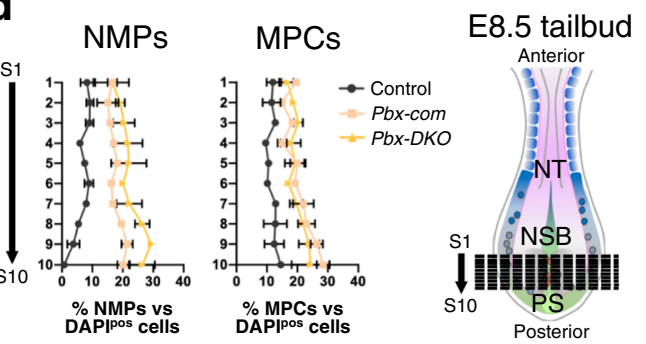

Fig. 2 Pbx mutant NMPs fail to acquire paraxial mesodermal fate, do not progress towards PSM, and accumulate in the tailbuds. a Confocal maximum intensity projection images of E8.5 tailbuds probed with T-BRA (green), SOX2 (red) and TBX6 (magenta) antibodies and counterstained with DAPI (grey) reveal the enlarged CLE and accumulation of T-BRA pos, SOX2pos, TBX6 ${ }^{\text {pos }}$ cells in Pbx-com and Pbx-DKO tailbuds. The cartoons on the right depict dorsal views of E8.5 control and Pbx mutant tailbuds, with NMPs represented as red dots and MPCs as blue dots. Scale bars: $50 \mu$ m. b Confocal transverse sections of control, Pbx-com and Pbx-DKO tailbuds through the caudal progenitor zone. Black dashed lines in the cartoon indicate the plane of sections. Representative sections S1 and S2 reveal accumulation of T-BRA/SOX2 double-positive (T-BRApos;SOX2pos) NMPs in Pbx mutants (yellow arrowheads). TBX6 staining shows reduced expression in PSM and co-localisation with NMP markers in Pbx-com and Pbx-DKO tailbuds (white arrowheads). The panel on the left summarises the molecular signature used to identify the different cell types along the path towards PSM. Scale bars: $30 \mu \mathrm{m}$. c Box plots assessing the proportion of T-BRA ${ }^{\text {pos; }}$ TBX6 ${ }^{\text {pos }}$ cells (MPCs) relative to DAPIpos cells (total cells) or to TBX6 ${ }^{\text {pos }}$ cells (PSM), and T-BRA ${ }^{\text {pos; }}$;OX2 ${ }^{\text {pos }}$ cells (NMPs) and TBX6 ${ }^{\text {pos }}$ cells (PSM) relative to DAPIpos cells. The box contains the 25th to 75th percentiles of the dataset, the centre line denotes the median value (50th percentile) and the whiskers extend from the smallest to the largest value. P-values were calculated by one-way ANOVA and Tukey's test for multiple comparisons and are indicated in the figure. $\mathbf{d}$ Distribution of NMPs and MPCs along the anterior/posterior axis shows accumulation of NMPs and MPCs in the posterior tailbud of $\mathrm{Pbx}$-com and Pbx-DKO embryos. Data are mean \pm s.e.m. Black dashed lines in the cartoon indicate the plane of sections. In c and d, the percentage of different cell types for each embryo was calculated from 10 independent sections equally distributed along the anterior/posterior axis. In $\mathbf{a}, \mathbf{b}$ and $\mathbf{d}$ : CLE caudal lateral epiblast, NSB node-streak border, PS primitive streak, PSM pre-somitic mesoderm, Som somites, NT neural tube, A anterior, $\mathrm{P}$ posterior, $\mathrm{D}$ dorsal, $\mathrm{V}$ ventral. 
a
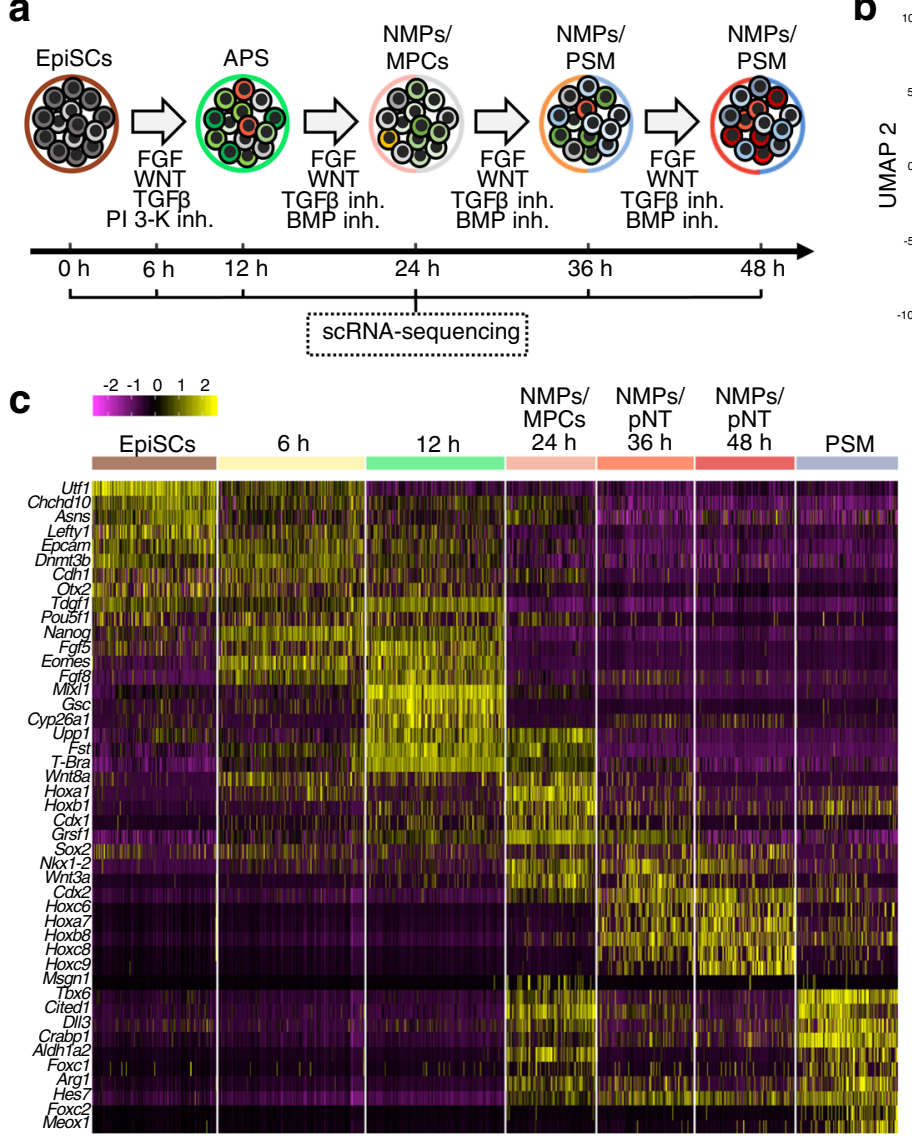

f

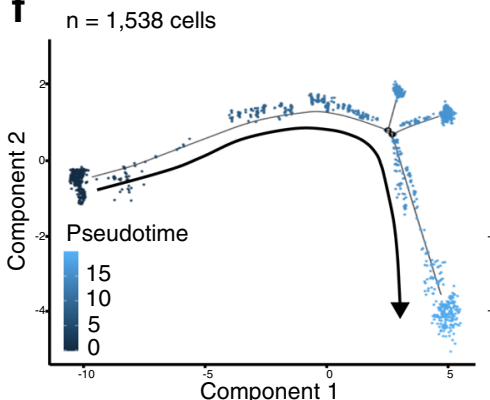

h
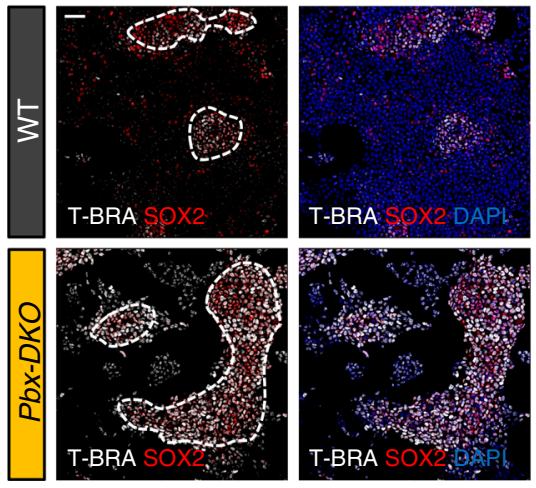

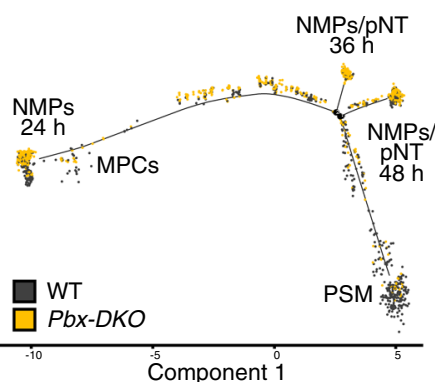

$48 \mathrm{~h}$

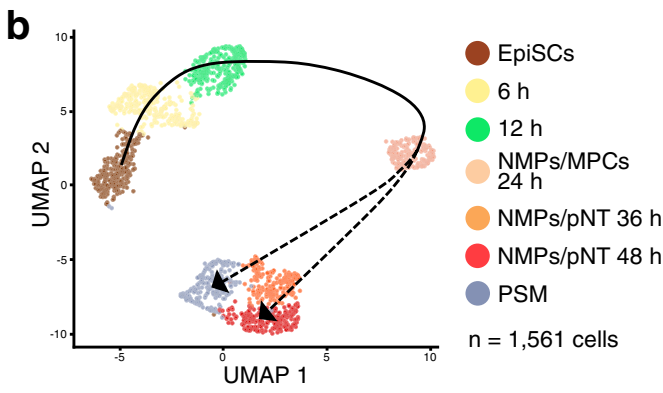

d

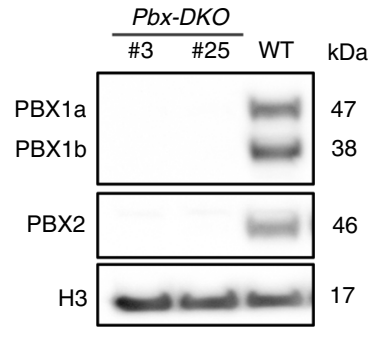

e

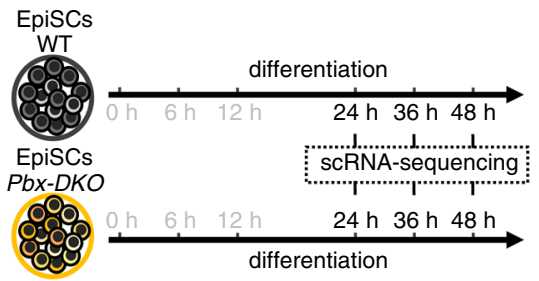

g

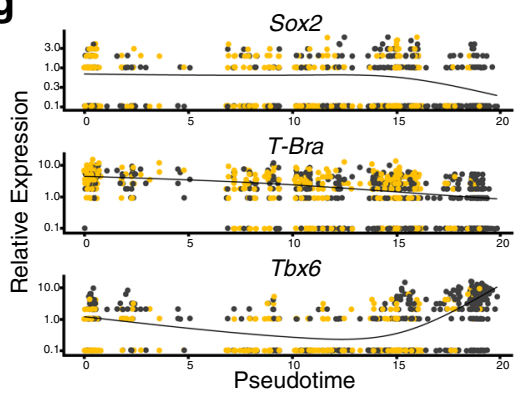

i

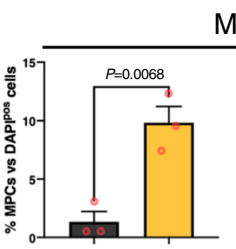

MPCs
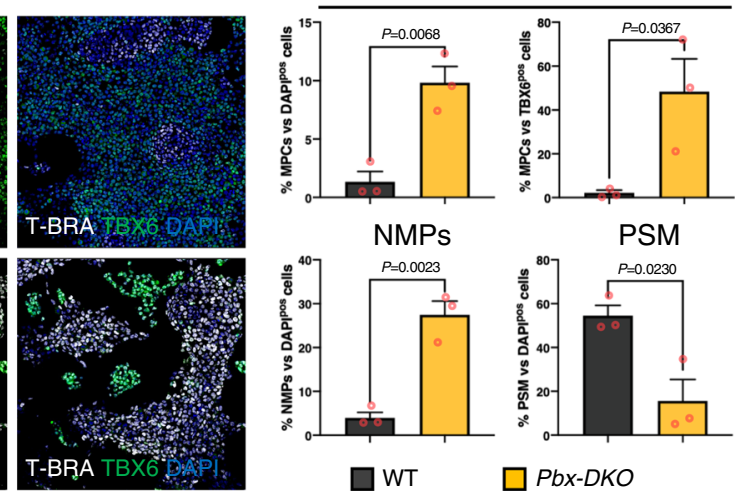

$\square$ WT

$P b x-D K O$
Data 5). In EpiSCs and at $6 \mathrm{~h}$, when HOX are absent, we identified the PBX3 and PREP1 binding motifs ${ }^{33}$, whereas at later time-points, once Hox genes are expressed, the HOX recognition sites featured prominently. Notably during the 12$24 \mathrm{~h}$ time window that includes the NMP-to-PSM transition, we identified LEF1 among the top consensus motifs nearby the PBX-bound regions. These data suggest that LEF1 and PBX might bind cooperatively to PSM genes, and provided a possible reason why $P b x-D K O$ cells cannot complete PSM maturation despite the presence of WNT ligands (Supplementary Fig. 2d). 
Fig. 3 Pbx mutant cells fail to acquire paraxial mesoderm identity in vitro. a Schematics of the in vitro protocol used to differentiate EpiSCs towards anterior primitive streak (APS) and pre-somitic mesoderm (PSM). b ScRNA-seq of in vitro PSM differentiation. Cells were harvested at different timepoints, as indicated in a. Unsupervised clustering and UMAP visualisation reveal temporally distinct populations of NMPs (orange-red), MPCs (light pink) and PSM (light blue). Individual cells are coloured according to annotated cluster identities. Reconstructed developmental trajectories are indicated with black dashed lines. c Heatmap showing expression of key genes along the path towards PSM. Cells acquire a NMP signature at $24 \mathrm{~h}$, and subsequently undergo progressive maturation towards PSM. NMPs present at 36-48 h express higher levels of posterior Hox genes (Hox6-9), consistent with the acquisition of more posterior axial identity. Colour bar indicates the intensity associated with normalised expression values. $\mathbf{d}$ Western blot analysis confirms absence of PBX1 and PBX2 proteins in the Pbx-DKO cell lines obtained by inactivating Pbx1 and Pbx2 in 129 EpiSCs with a CRISPR/Cas9 approach. e Schematics of wild-type (WT) and Pbx-DKO EpiSCs differentiation towards PSM. The time-points selected for scRNA-seq analyses are highlighted in black. f Monocle's pseudotemporal ordering of WT (black) and Pbx-DKO cells (yellow) spanning the NMP-to-PSM transition (24-48 h), showing inability to generate PSM and increased number of NMPs/MPCs caused by loss of PBX. $\mathbf{g}$ Pseudotime analysis of PSM differentiation showing distribution of WT (black) and Pbx-DKO cells (yellow) expressing the NMP genes Sox2 and T-Bra and the PSM marker Tbx6. h Representative immunofluorescence staining

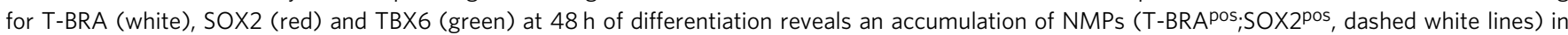
Pbx-DKO cells. Scale bar: $50 \mu \mathrm{m}$. i Histograms representing the percentage of progenitors in WT (black) and Pbx-DKO cells (yellow) at $48 \mathrm{~h}$ of differentiation. Upper panel: percentage of MPCs (T-BRApos;TBX6pos) relative to DAPIpos cells (total cells) or to TBX6 pos cells (PSM). Lower panel: percentage of NMPs (T-BRApos;SOX2pos) and PSM (TBX6 ${ }^{\text {pos }}$ ) cells relative to DAPIpos cells. Data are mean \pm s.e.m. $P$-values indicated in the figure were calculated by two-tailed unpaired $t$-test $(n=3$ biological replicates)

PBX proteins are interpreters of LEF1-mediated WNT signalling. Like PBX, the WNT effectors (TCFs/LEF1) exhibit low binding specificity ${ }^{34}$. Therefore, the observation that PBX and LEF1 could be part of a common regulatory module supported the hypothesis of a PBX-LEF1 DNA-binding cooperativity at PSM genes. LEF1 and TCF7 are the most prominent WNT effectors involved in paraxial mesoderm development, but while the latter is broadly expressed in the tailbud, LEF1 is specifically localised in PSM and posterior somites ${ }^{35}$ (Fig. 5a). Notably, LEF1 expression is expanded in E8.5 Pbx-DKO tailbuds, suggesting that the $P b x$ mutant phenotypes are not due to a reduction in LEF1 levels (Fig. 5a). To assess whether PBX and LEF1 TFs cooperatively control common transcriptional targets, we exploited our in vitro differentiation system, and performed ChIP-seq for LEF1. We found that LEF1 is de novo and progressively recruited to chromatin between 12 and $24 \mathrm{~h}$, concomitantly with the formation of MPCs (Fig. 5b). Remarkably, LEF1 recruitment is abolished in mutant cells (Fig. 5b), despite sustained LEF1 expression (Fig. 5a and Supplementary Fig. 5a). By integrating our ChIP-seq datasets for PBX and LEF1 (Fig. 5c and Supplementary Data 6) and RNA-seq of WT and Pbx-DKO cells at $24 \mathrm{~h}$ (Supplementary Fig $5 \mathrm{~b}$ and Supplementary Data 6), we observed that over $60 \%$ of the LEF1 targets (including Sox2, T-Bra, Cdx2, Msgn 1 and Aldh1a2) were co-bound by PBX (Fig. 5c) and misregulated in $P b x-D K O$ (Supplementary Fig. 5b). As expected, GO-term analyses underscored targets associated with WNT activity and somitic features (Fig. 5d). We observed that while the occupancy of PBX and LEF1 occurs at variable distances on different regulatory regions, the majority (61\%) of the LEF1 ChIP-seq peaks falls in close proximity to the PBX peaks (Supplementary Fig. 5c). ATAC-seq analyses of PBX-LEF1 common targets revealed that chromatin accessibility at LEF1bound regions decreased for downregulated genes (Supplementary Fig. 5d) and remained unchanged for upregulated genes (Supplementary Fig. 5e), as illustrated for Msgn1, Aldh1a2, Sox2 and Cdx2 (Fig. 5e). These data demonstrate that PBX proteins authorise WNT-dependent transcription by making PSM genes accessible to LEF1. Instead, in the absence of PBX, the inability to activate the PSM fate programme triggers the feedforward mechanisms sustaining NMP expansion.

Distinct PBX complexes are sequentially recruited to the Msgn1 regulatory region. To establish how the PBX-LEF1 module operates, we focussed on the master regulator of the paraxial mesoderm fate, Msgn1, which controls both cell migration and differentiation ${ }^{10}$. Undifferentiated progenitors accumulate in Msgn1 mutant tailbuds comparably to $P b x-D K O$ embryos $^{10,14}$. Notably, Msgn 1 expression was lost in Pbx-com embryos (Fig. 6a) and strongly downregulated in $\mathrm{Pbx}-\mathrm{DKO}$ cells upon PSM differentiation (Fig. 6b). Moreover, the MSGN1 target Snai $1^{10}$ was downregulated in E8.5 Pbx-DKO and Pbx-com PSM (Supplementary Fig. 6a), suggesting that epithelial-tomesenchymal transition (EMT) and cell migration are affected in absence of PBX. To assess the cell migratory capacity of WT and mutant MPCs, we visualised PSM differentiation in timelapse videos (Supplementary Movies 1 and 2 and Supplementary Fig. 6b). We observed that WT cells leave the NMP zone upon differentiation, while $P b x-D K O$ cells fail to migrate and accumulate in the expanding progenitor area. Lentiviral re-expression of Msgn1 in $P b x-D K O$ cells during differentiation resulted in downregulation of NMP genes and upregulation of PSM genes (Supplementary Fig. 6c). These data confirmed that PBX-LEF1 direct PSM development and EMT by controlling Msgn 1 expression.

Msgn1 is the earliest expressed paraxial mesoderm gene and is tightly regulated in vivo and in vitro, being transcribed in the 12-36 $\mathrm{h}$ window corresponding to the NMP-to-PSM transition (Fig. 6b). ChIP-seq data showed that PBX and LEF1 are dynamically recruited to the Msgn 1 promoter, mirroring $M s g n 1$ temporal expression, and LEF1 occupancy is lost in $P b x-D K O$ cells (Fig. 6c).

To identify the factors that temporally recruit LEF1 on Msgn1 promoter, we focussed on PBX and their combinatorial logic ${ }^{36}$. Based on the temporal expression of HOX and TALE family members, we identified several candidates such as the heterodimerizing partner Meis2 and group-1 Hox genes (Hox-1) (Supplementary Fig. 7a) that are expressed during the period of PBX occupancy at the Msgn1 promoter. In addition, a second PBX-interacting factor, PREP1, is expressed earlier than Msgn1 (Fig. 6d). Considering the MPC-specific enrichment of Pbx1, Meis2 and Hox-1 (Fig. 6e) these proteins represent the best candidates for regulating Msgn1. We hypothesised that two putative complexes would be sequentially recruited, with PREP1/ $\mathrm{PBX}$ binding prior to the PBX/MEIS2/HOX-1 complex. Consistent with this idea, ChIP-qPCR showed that PREP1 is recruited to Msgn1 first, at $12 \mathrm{~h}$, while MEIS2 and PBX are recruited later, at $12-24 \mathrm{~h}$ (Fig. 6f). Examining the Msgn1 regulatory region, we identified a PBX/HOX-1 responsive site ${ }^{37}$ within the region of PBX occupancy (Fig. 6g and Supplementary Fig. 7b). Electrophoretic Mobility Shift Assay (EMSA) with either in vitro- 
a

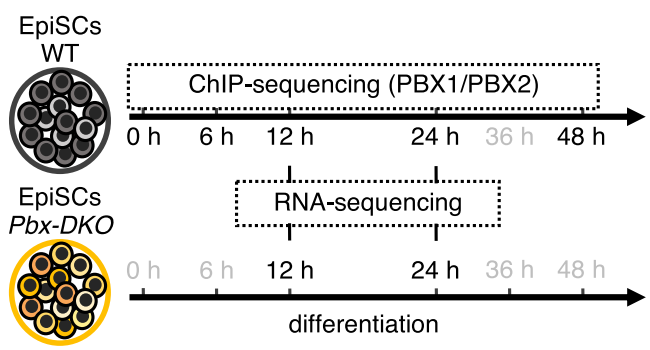

b

Genes downregulated in $\mathrm{Pbx}-\mathrm{DKO}$ at $24 \mathrm{~h}$

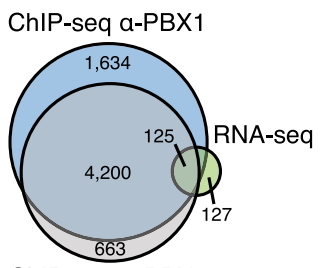

ChIP-seq a-PBX2
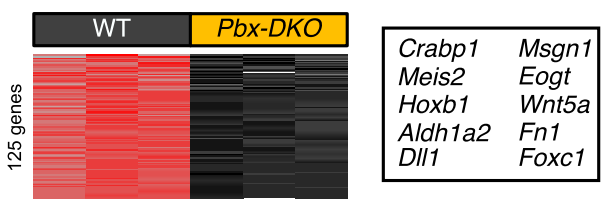

\section{C}

Genes upregulated in $P b x-D K O$ at $24 \mathrm{~h}$
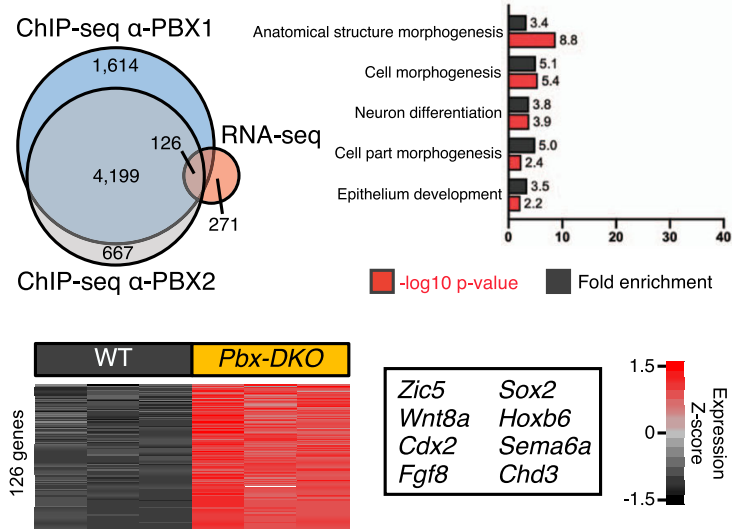

d
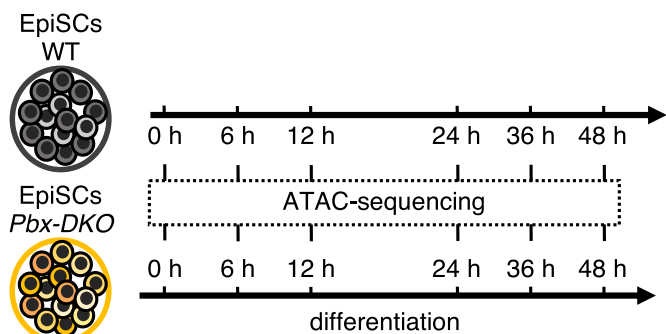

translated proteins or $24 \mathrm{~h}$ nuclear extracts highlighted the PBX/ MEIS2/HOX-1 ternary complex formation on the oligonucleotide containing the $\mathrm{PBX} / \mathrm{HOX}-1$-binding site (Msgn1-P2) but not on its mutated variant (Msgn1-P2-M) (Fig. 6h). In agreement with the sequential recruitment to the Msgn1 regulatory region, we found that while the assembly of PBX/MEIS2 on Msgn1-P2 e

\section{Genes downregulated in $P b x-D K O$ at $24 \mathrm{~h}$}

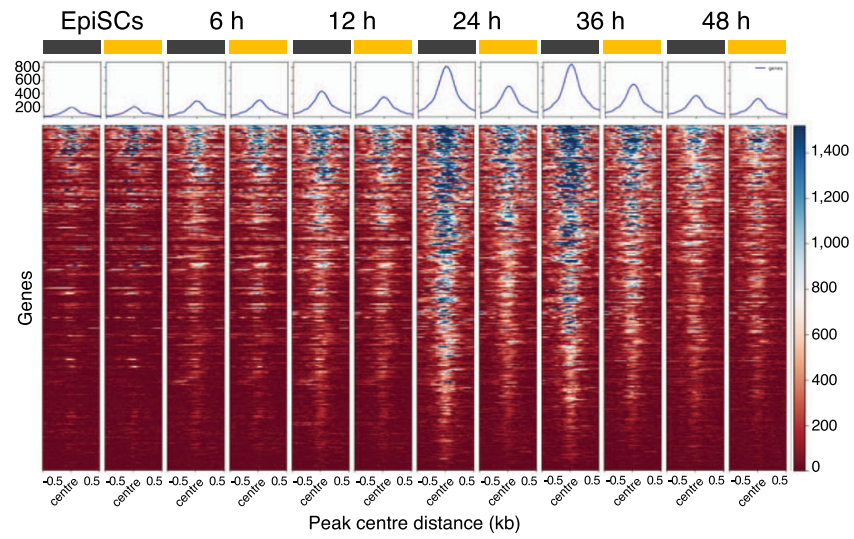

f

Genes upregulated in $P b x-D K O$ at $24 \mathrm{~h}$

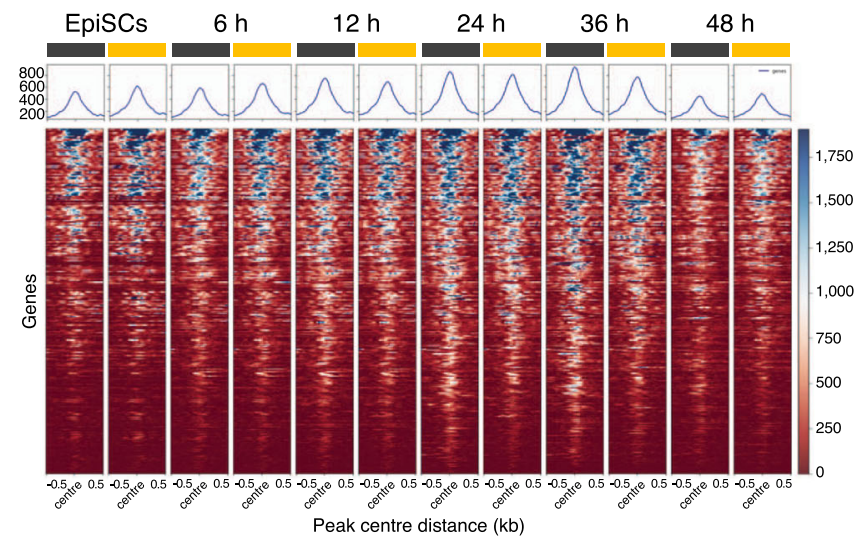

g

\begin{tabular}{|c|c|c|c|}
\hline Time-point & Enriched Motif & Factor & $P$ value \\
\hline \multirow{2}{*}{ EpisCs } & 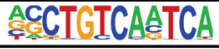 & $\mathrm{PBX} 3$ & $1 \mathrm{e}^{-1,640}$ \\
\hline & 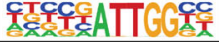 & $\mathrm{NFY}$ & $1 e^{-556}$ \\
\hline \multirow{2}{*}{$6 \mathrm{~h}$} & IGATTGACAGE & PREP1 & $1 \mathrm{e}^{-1,376}$ \\
\hline & CCAAIFGGAE & NFY & $1 e^{-231}$ \\
\hline \multirow{4}{*}{$12 \mathrm{~h}$} & ACCTGTCAETCA & PREP1 & $1 e^{-2097}$ \\
\hline & CCTATTGG & $\mathrm{NFY}$ & $1 e^{-438}$ \\
\hline & GATTTATATG & HOXA9 & $1 \mathrm{e}^{-196}$ \\
\hline & C्CTTTGATCT & LEF1 & $1 e^{-54}$ \\
\hline \multirow{4}{*}{$24 \mathrm{~h}$} & TGATTGATCG & PBX2 & $1 \mathrm{e}^{-2806}$ \\
\hline & 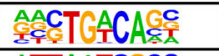 & MEIS1 & $1 e^{-962}$ \\
\hline & 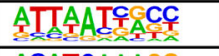 & HOXC9 & $1 \mathrm{e}^{-575}$ \\
\hline & ACATCAAAGG & LEF1 & $1 e^{-262}$ \\
\hline \multirow{4}{*}{$48 \mathrm{~h}$} & TGAITGACCGG & PBX2 & $1 \mathrm{e}^{-2,276}$ \\
\hline & CCTGTCAG & MEIS2 & $1 e^{-652}$ \\
\hline & 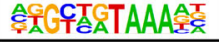 & CDX4 & $1 e^{-565}$ \\
\hline & TEATTGGCTE्र & NFY & $1 \mathrm{e}^{-274}$ \\
\hline
\end{tabular}

depended on the presence of HOX-1 (Supplementary Fig. 7c), the PREP1/PBX heterodimers could bind in its absence (Supplementary Fig. 7d). These binding properties suggest that the expression of HOX-1 drives the assembly of a new complex at the Msgn 1 promoter that would be responsible for LEF1 occupancy and WNT-mediated activation. Consistent with this idea, affinity 
Fig. 4 PBX proteins occupy regulatory regions associated with PSM genes and remodel chromatin accessibility. a Schematics of EpiSC differentiation towards PSM. The time-points selected for ChIP-seq/RNA-seq analyses are highlighted in black. $\mathbf{b}$ Top: overlap of downregulated genes in Pbx-DKO cells (RNA-seq) and genes associated with PBX-occupied regions at $24 \mathrm{~h}$ (ChIP-seq). GO-term enrichment analysis displays significant overrepresentation of terms associated with mesoderm and somite development (Binomial test with Bonferroni correction). Bottom: heatmap showing RNA-seq expression Z-scores computed for 125 downregulated PBX targets in Pbx-DKO (yellow) compared to WT cells (black). Representative markers are indicated. c Top: overlap of upregulated genes in Pbx-DKO cells (RNA-seq) associated with PBX-occupied regions (ChIP-seq) at $24 \mathrm{~h}$. GO-term enrichment analysis shows correlation with terms associated with neurectoderm and embryonic development (Binomial test with Bonferroni correction). Bottom: heatmap displaying RNA-seq expression Z-scores computed for 126 upregulated PBX targets in Pbx-DKO (yellow) compared to WT cells (black). Representative markers are indicated. In $\mathbf{b}$ and $\mathbf{c}$, significance was assessed by DEseq 2 on the basis of two-sided Wald test with Benjamini-Hochberg adjusted $P$-values $(P \leq 0.05$, foldchange $\geq 1.5$ ). $n=3$ biological replicates. $\mathbf{d}$ Schematics of EpiSC differentiation towards PSM illustrating the time-points selected for chromatin accessibility analysis (ATAC-seq). e Heatmap of chromatin accessibility of WT (black) and Pbx-DKO cells (yellow) at PBX-occupied regions that are associated with downregulated genes in Pbx-DKO along PSM differentiation, showing reduced accessibility in Pbx-DKO cells at 24 and $36 \mathrm{~h}$. $\mathbf{f}$ Heatmap of chromatin accessibility at PBX-bound regions associated with upregulated genes in Pbx-DKO compared to WT cells. Chromatin accessibility is comparable between WT (black) and Pbx-DKO cells (yellow). The scale represents normalised counts (RPKM) for ATAC-seg peak signals \pm 500 bp around the centre of the peak in $\mathbf{e}$ and $\mathbf{f}$. $\mathbf{g}$ Homer de novo motif analysis of 200 bp summit regions along differentiation reveals changing in binding sites enrichment at different time-points, highlighting the variety of PBX-binding partners during PSM differentiation. Only the most relevant binding motifs are shown for each timepoint. See Supplementary Data 5 for the complete list.

binding assays confirmed that the PBX/MEIS2/HOX-1 complex has higher affinity for Msgn1-P2 than PBX/PREP1 complexes, suggesting that it could compete for binding once HOX-1 is expressed (Supplementary Fig. 7e-g). Moreover, we observed that changes in the accessibility of Msgn 1 regulatory regions were correlated with Hox-1 expression, and concurrent with PBX-LEF1 recruitment. Accordingly, in $P b x-D K O$ the accessibility of the loci containing the $\mathrm{PBX} / \mathrm{HOX}$ and LEF1-binding sites was reduced at $24 \mathrm{~h}$ (Fig. 6i), highlighting a potential role of $\mathrm{PBX} / \mathrm{HOX}$ complexes to nucleate their own binding and their ability to destabilise the nucleosomes. These data uncover a hierarchical TF-binding network on the Msgn 1 locus, but do not conclusively demonstrate that $\mathrm{PBX} / \mathrm{HOX}$ are directly involved in chromatin remodelling, prompting us to assess the recruitment of additional pioneer factors.

TALE complexes direct chromatin remodelling at the Msgn1 regulatory region, synergising with T-BRA. T-BRA is a pioneer factor essential for priming mesodermal genes ${ }^{38}$. ChIP-qPCR analyses showed that T-BRA is already recruited at $12 \mathrm{~h}$ to the LEF1binding region of Msgn1, and its occupancy decreases over time (Fig. 7a). Interestingly, T-BRA occupancy is not abrogated in $P b x$ $D K O$ (Fig. 7a), suggesting that the binding of T-BRA on Msgn 1 is not sufficient to drive paraxial mesoderm differentiation and that PBX complexes remodel the chromatin independently of T-BRA.

To establish the direct consequence of the binding of PBX complexes on chromatin accessibility and their effects on LEF1 recruitment, we employed CRISPR/Cas9 technology to generate lines carrying the $p M s g n 1$ point mutations ( $p M s g n 1-m u t)$ studied by EMSA (Fig. 6g, h and Supplementary Fig. 8a, b), preventing the binding of PBX complexes to the Msgn1 promoter but allowing their recruitment to other targets. RT-qPCR analyses showed that the expression of Msgn1 and its downstream targets were affected in pMsgn1-mut cells (Fig. 7b), and PSM differentiation was impaired (Fig. 7c), thus recapitulating at least in part the $P b x-D K O$ phenotype. However, the mesodermal phenotype of pMsgn1-mut lines was exacerbated, as shown by the absence of T-Bra, the paraxial mesodermal gene Tbx6, and the EMT markers Cdh2 and Snail. ChIP-qPCR revealed that in pMsgn1-mut neither PBX nor LEF1 were recruited to Msgn1 (Fig. 7d). Interestingly, the recruitment of T-BRA was also affected (Fig. 7d) in pMsgn1-mut, and ATAC-seq analyses showed that both LEF1 and PBX/HOX-binding regions were inaccessible in pMsgn1-mut cells (Fig. 7e), unequivocally confirming the role of PBX complexes as pioneer factors and modulators of chromatin accessibility. Furthermore, the reduced recruitment of T-BRA reveals that target gene selection specificity is achieved through the collaborative effort of multiple pioneer TFs.

In conclusion, paraxial mesoderm induction relies on a WNTHOX integrated code that is coordinated by the TALE complexes, the WNT-effector LEF1 and the synergistic activity of T-BRA. While T-BRA broadly assesses mesodermal genes, TALE/HOX-1 interpret WNT signalling response by specifically recruiting LEF1 at the PSM genes, resulting in paraxial mesoderm differentiation via a Msgn1-mediated transcriptional programme.

\section{Discussion}

In this work, we dissected how TALE complexes control the GRNs that alter the NMP response to WNT signalling and activate the paraxial mesoderm programme. We established that TALE/HOX and WNT signalling work through a common genetic code that simultaneously represses NMP genes and activates the PSM differentiation programme. We found that switching of the PBX-binding partners result in TALE/HOX high-affinity complex formation that destabilises the nucleosomes at WNT-responsive elements enabling de novo recruitment of the WNT-effector LEF1, thereby challenging the consolidated model of WNT-mediated response centred on the pre-bound activity of TCFs/LEF.

This concerted TALE-LEF1 activity represses NMP genes (like Sox2, T-Bra and $C d x 2$ ), ensuring silencing of the neural and NMP transcriptional programmes in PSM-fated cells. Concurrently, TALE-LEF1 synergistically drive the expression of PSM genes including the master regulator Msgn1, which in turn controls EMT and the migration of MPCs from the progenitor zone to the anterior PSM. Loss of the TALE activity results in the accumulation of progenitors trapped in a NMP/MPC state that ultimately causes an enlarged tailbud and impaired somitogenesis (Fig. 8a).

In vertebrates, Hox genes are collinearly expressed during axial elongation, establishing the HOX code with a spatio-temporal gradient along the anterior-posterior axis ${ }^{19,20,39}$. This sequential activation provides NMPs with positional information and prompts MPCs to progressively adopt a more posterior identity ${ }^{11}$, ensuring that cell fate and position are intrinsically linked. Our data suggest that group-1 HOX are also indispensable for the initial specification of MPCs and their migration to form PSM; thereby balancing the residence time in the progenitor zone and the flux of PSM patterning the trunk region. In Pbx mutants, $5^{\prime}$ Hox genes are overexpressed (Supplementary Fig. 1h) in NMP/ MPCs, suggesting the existence of progenitors with a posterior identity and thereby a potential posteriorisation of the PSM, in 
a
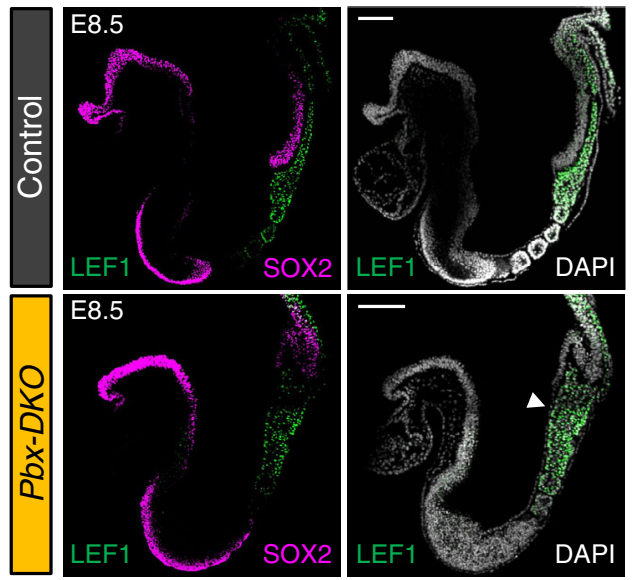

C

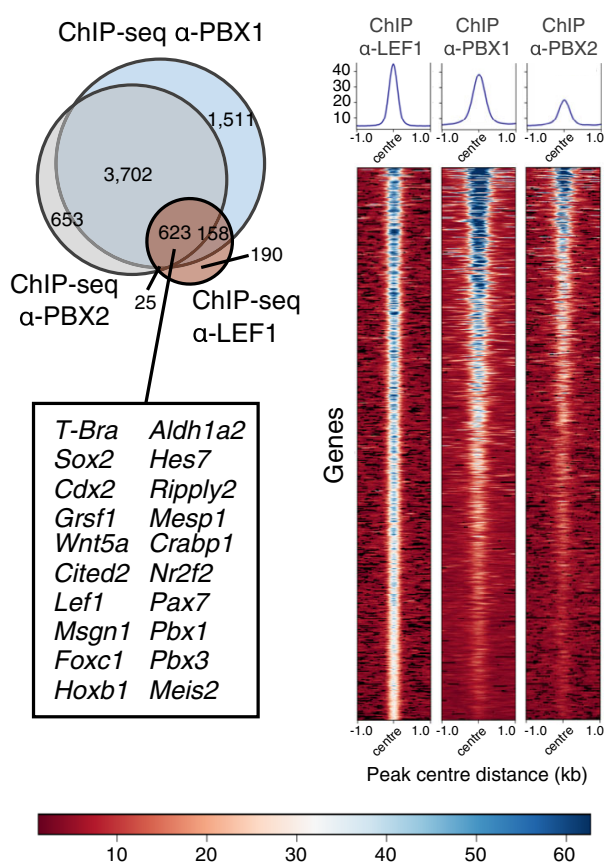

d

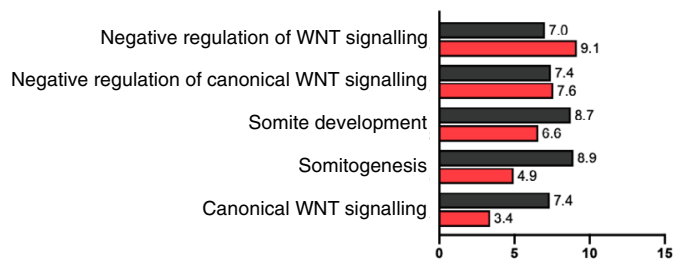

-log10 P-value b

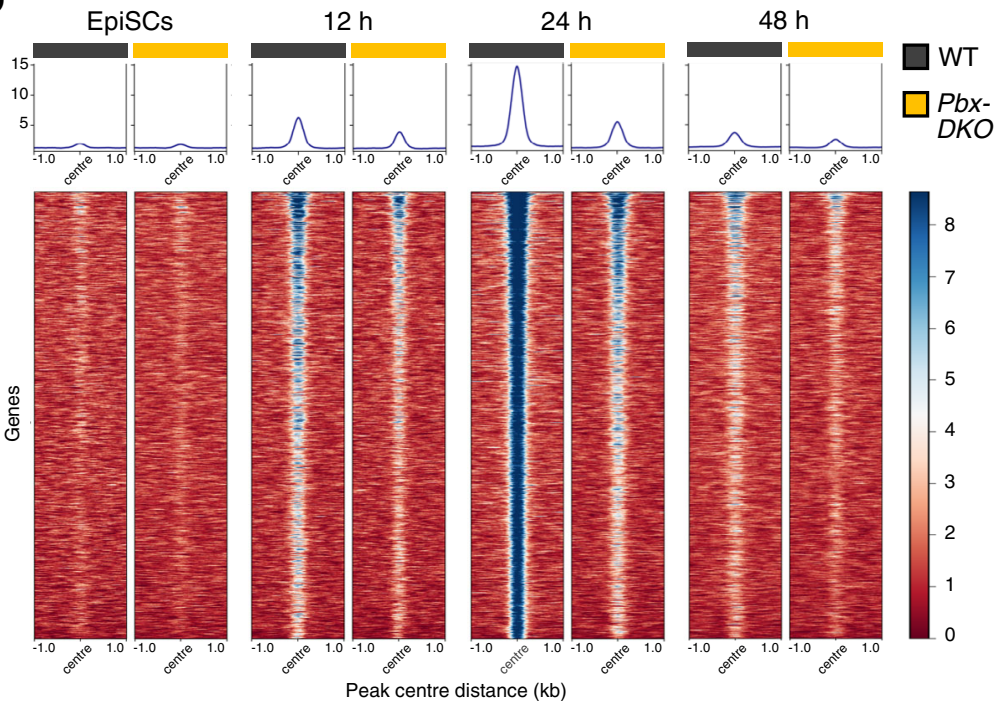

e
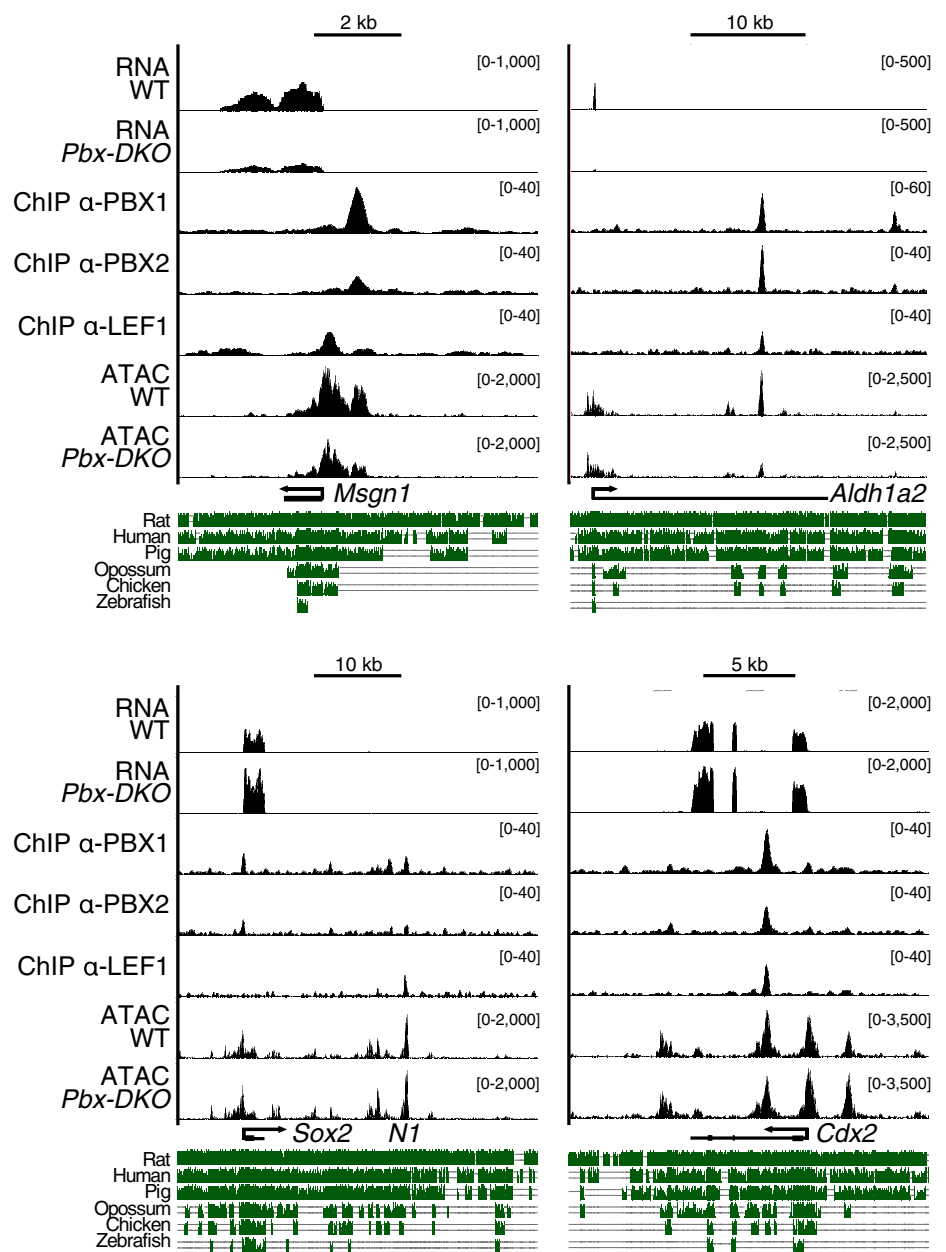

agreement with the observation of a rostral shift of the axial Hox gene expression in the $P b x$-com mutants ${ }^{25}$.

In addition to providing HOX with binding specificity ${ }^{37}$, PBX have also been indicated as pioneer factors ${ }^{29,31}$. However, a key unanswered question is how such broadly expressed TFs can exquisitely open lineage-specific loci in a space and timedependent manner. Exploiting the cooperative interactions among TALE and HOX proteins, and focussing on the tightly regulated spatio-temporal expression of Msgn1, we uncovered the sequential recruitment of TALE factors generating, at least in vitro, a high-affinity (PBX/MEIS2/HOX-1) ternary complex that could promote chromatin accessibility of the Msgn1 promoter (Fig. 8b). While the binding of the PBX/MEIS2 complex occurs concomitantly with increased chromatin accessibility and LEF1 recruitment, the low-affinity PBX/PREP1 dimers are recruited earlier, suggesting that they could be important to scan the DNA or increase the local concentration of PBX proteins, generating the TALE hubs that assist the loading of the TALE/ 
Fig. 5 PBX complexes remodel the chromatin environment for LEF1 recruitment. a Representative immunofluorescence staining for LEF1 (green) and SOX2 (magenta) on sagittal sections of E8.5 mouse embryos, revealing specific localisation of LEF1 in PSM and posterior somites of control and Pbx-DKO embryos. LEF1 expression is bloated in the tailbud of Pbx-DKO (white arrowhead). DAPI was counterstained in grey. Scale bars: $100 \mu \mathrm{m}$. b Heatmap of LEF1 ChIP-seq signals of WT (black) and Pbx-DKO cells (yellow) reveals PBX crucial role in LEF1 recruitment, as demonstrated by the extensive drop of LEF1 occupancy in Pbx-DKO cells along differentiation. c Left: overlap of genes bound by PBX1 (light blue), PBX2 (grey) and LEF1 (brown). A representative subset of target genes co-bound by PBX and LEF1 is reported in the lower box. Right: heatmap of tag densities of LEF1, PBX1 and PBX2 ChIP-seq peaks at the co-bound regions identified at $24 \mathrm{~h}$ of differentiation. In $\mathbf{b}$ and $\mathbf{c}$, the scale represents normalised counts (RPKM) for ChIP-seq peak signals $\pm 1 \mathrm{~kb}$ around the centre of the peak. $\mathbf{d}$ GO-term enrichment analysis of genes bound by PBX and LEF1 displays significant overrepresentation of terms associated with WNT signalling and somite development (Binomial test with Bonferroni correction). e RNA-seq, ChIP-seq and ATAC-seq coverage tracks for PBX-LEF1-activated (Msgn1, Aldh1a2) and repressed (Sox2, Cdx2) target genes. Threshold of vertical viewing range of data based on RPKM values is noted. Conservation across vertebrates is indicated in green.

HOX complex ${ }^{40}$. The observation that PBX are pre-bound on regulatory regions prior to gene activation ${ }^{29-31}$ has been used as proof for their pioneering activity. However, there are no data conclusively showing that they unlock closed chromatin. The pioneer activity of TFs is usually determined by measuring reduced chromatin accessibility in wild-type and knockout cells ${ }^{38}$, that however can also be caused via indirect mechanisms. Here, using precise CRISPR-mediated mutagenesis, we unequivocally demonstrate that point mutations affecting the binding of PBX/ HOX to the endogenous Msgn1 promoter reduce chromatin accessibility and LEF1 recruitment, indicating that PBX directly modulate chromatin accessibility and context-dependent WNT responses.

Analyses of PBX and LEF1 occupancy revealed that the distance between them is flexible and varies at different regulatory regions. This arrangement could support cooperative binding, allowing the specific recruitment of TFs that further stabilise the nucleosome-depleted regions triggered by the $\mathrm{PBX}$ complexes. Thus, changes in chromatin accessibility initiated by the PBX complexes could mediate recruitment of additional factors, including ATP-dependent chromatin remodelling factors. PBX/ HOX could interact with histone modifying complexes like CBP, TrxG or HDAC and PcG proteins ${ }^{41}$ or may affect chromatin conformation via recruitment of histone variants ${ }^{42}$. TF-binding sites located in closed chromatin could provide an additional layer of specificity by restricting the access to highly specific PBX/ HOX complexes ${ }^{43,44}$. Interestingly, the NFY-binding site appeared among the most enriched motifs identified within the PBX-bound regions at all time points of differentiation ${ }^{45}$. In zebrafish, NFY has been demonstrated to directly interact with the TALE proteins to promote access to enhancers, suggesting that it could play a similar role in paraxial mesoderm differentiation. For all these reasons, it is plausible that chromatin landscape changes are driven by combined effects on DNAhistone affinity and recruitment of nucleosome remodelling factors.

Intriguingly, the TALE-WNT integrated code is conserved in the regulatory regions of PSM genes within eutherians where axial elongation relies on expanding NMPs, but is absent in lower vertebrates where PSM maturation is supported by depleting progenitors $^{6,46}$. Of note, the TALE-WNT integrated motif is present at the Sox2 enhancer $\mathrm{N1}^{47}$ that is exclusively associated with expansion of NMPs in amniotes. Thus, during vertebrate evolution, WNT, TALE and HOX proteins could have been coopted by eutherians to balance expansion and differentiation of paraxial mesoderm progenitors for axial elongation.

Prevailing models of WNT activity suggest that TCFs/LEF work through a transcriptional switch, binding and repressing genes in unstimulated conditions and promoting transcription upon WNT induction ${ }^{34}$. Here we observed that during paraxial mesoderm induction, LEF1 is de novo recruited when WNT signalling is induced and when the PSM genes become accessible.
Thus, in this context, the competence for WNT signalling response is established during the transition of NMPs to PSM. This alternative mechanism supports the concept that distinct modes exist to prime chromatin accessibility and lineage choice. Crucially, we found that signalling response is dictated by TFs unlocking the WNT-responsive elements rather than by WNT ligand dosage, questioning the current concentration-dependent morphogen model ${ }^{48}$.

Given that WNT/LEF1 signalling is important for homeostasis and regeneration of the skin ${ }^{49}$, it will be compelling to address if the TALE/HOX motif also unlocks the regenerative transcriptional programmes in the skin, thus having potential therapeutic applications. Furthermore, abnormal activity within this pathway is linked to hyperproliferation and EMT-driven metastatic processes $^{50}$. In this context, it will be important to establish if aberrations in TALE-WNT integrated codes could also be associated with carcinogenesis and cancer invasion ${ }^{51}$.

Finally, the observation that TALE complexes collaborate with the pioneer factor T-BRA ${ }^{38}$ provides a temporal code for WNTmediated response. While T-BRA has a pan-mesodermal role, the TALE complexes provide specificity towards the paraxial mesodermal fate. Hence, conventional cooperativity can be extended to pioneer factor clusters that synergistically generate a permissive chromatin landscape and collectively operate as key determinants of lineage specification. Given that the GRNs governing paraxial mesoderm differentiation ${ }^{17}$ are conserved between human and mouse, these findings can be used to improve the current protocol of hESCs differentiation.

Altogether, this work uncovers a cooperative TALE/HOXWNT integrated code unlocking the spatio-temporal GRNs that control axial extension. During axial elongation, similar modules could also be employed by 5 ' $\mathrm{HOX}$ proteins to further fine-tune WNT signalling response to provide MPCs with a more posterior fate. Thus, integrating Hox collinearity and WNT signalling on a common genomic element may represent a powerful strategy that confers mesodermal progenitors with a specific spatio-temporal identity.

\section{Methods}

Cell Lines. EpiSC lines were maintained in six-well cell-culture plates (Corning, 3506) pre-coated with $15 \mu \mathrm{g} / \mathrm{ml}$ human Fibronectin (Merck Millipore, FC01015) in N2B27 medium (prepared as described in Ying et al. ${ }^{52}$, supplemented with $20 \mathrm{ng} / \mathrm{ml}$ Activin A, $10 \mathrm{ng} / \mathrm{ml}$ bFGF and $1 \mu \mathrm{M}$ XAV939 (EpiSC medium). All lines used in this study were routinely karyotyped and tested for mycoplasma.

Mice. The mutant alleles used in this study were previously described: $P b x 1$ conditional allele in Koss et al..$^{53}$ and $\mathrm{Pbx}^{-/-}$mice in Selleri et al. ${ }^{54}$ The Cre deleter strain, Sox $2^{C r e}$ knock-in mouse ${ }^{55}$, was utilised for $P b x 1$ inactivation in the inner cell mass. All animals used in this study were maintained in laboratory animal housing facilities at macroenvironmental temperature and humidity ranges of $20-24^{\circ} \mathrm{C}$ and $45-65 \%$, respectively, with a $12 / 12 \mathrm{~h}$ light/ dark cycle. Embryos were dissected at E8.5/E9.0 and genotyped by PCR as described in Koss et al. ${ }^{53}$. All animal work was carried out in accordance with European legislation. All work was authorised by and carried out under the 
a

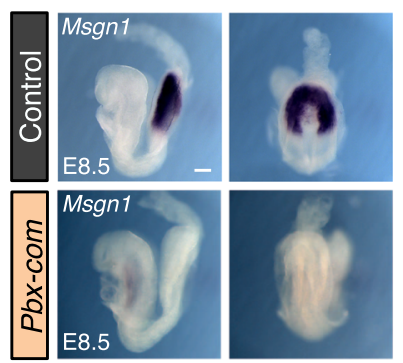

b

b $\square \mathrm{WT} \quad \square$ Pbx-DKO

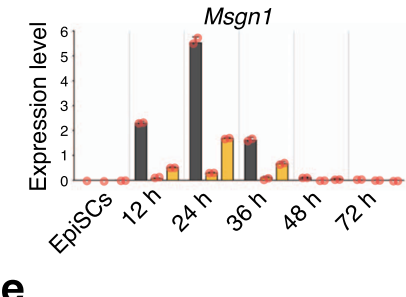

Average expression: $\begin{array}{rrrrrr}-1.0 & -0.5 & 0 & 0.5 & 1.0 & 1.5\end{array}$

$\%$ of cells expressing
marker genes:

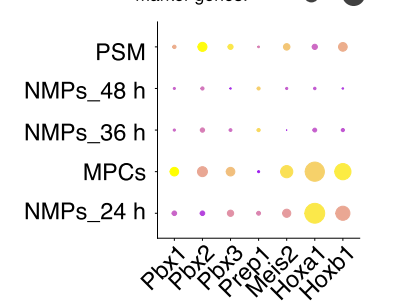

C

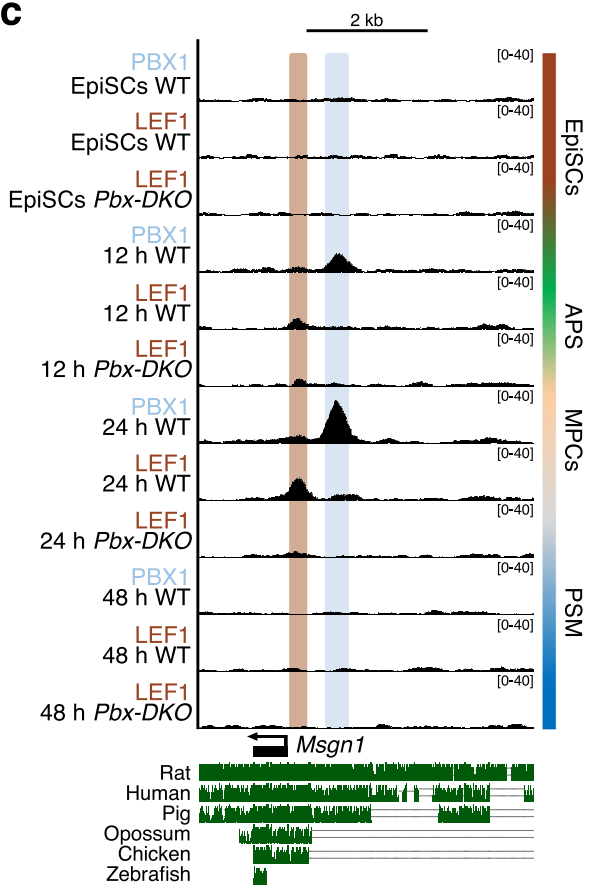

f

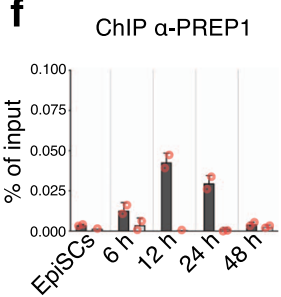

ChIP a-PBX1

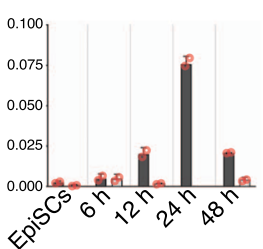

d

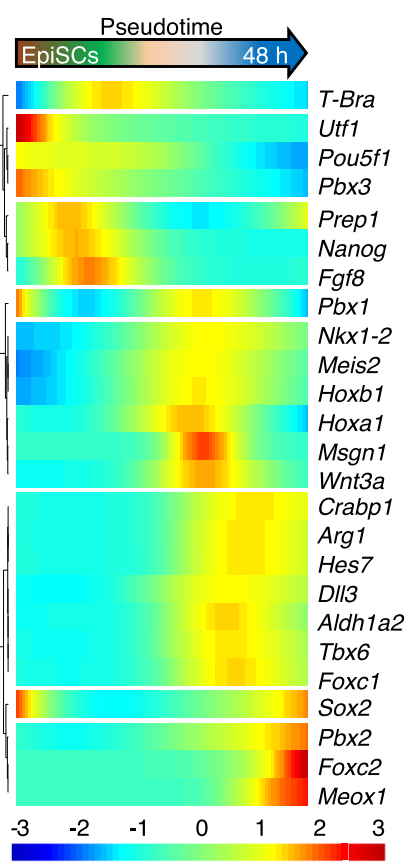

g

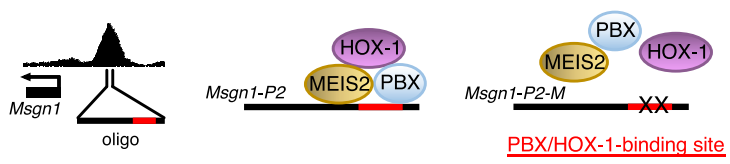

Msgn1-P2 CAGGAGCTCACAAGTGTACCTGGCTGCCAGTTAGCCTGATGAATGTCTT Msgn1-P2-M CAGGAGCTCACAAGTGTACCTGGCTGCCAGTTAGCCTcgTGcoTGTCTT

h

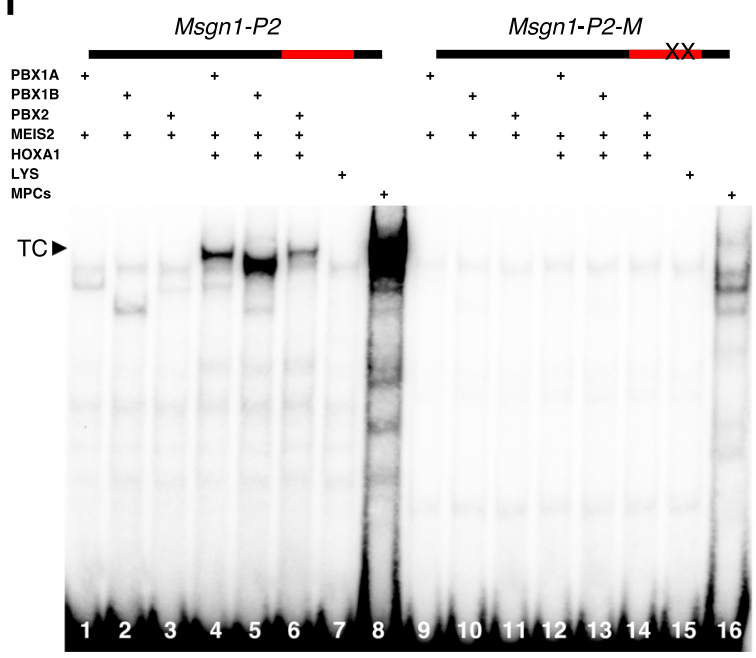

ChIP a-MEIS2

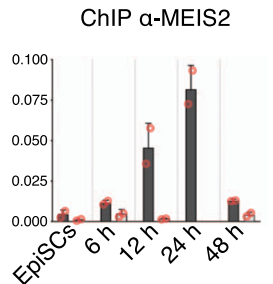

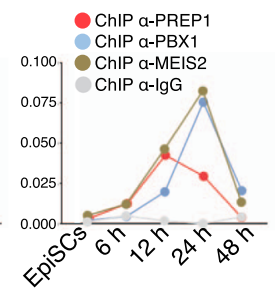

i

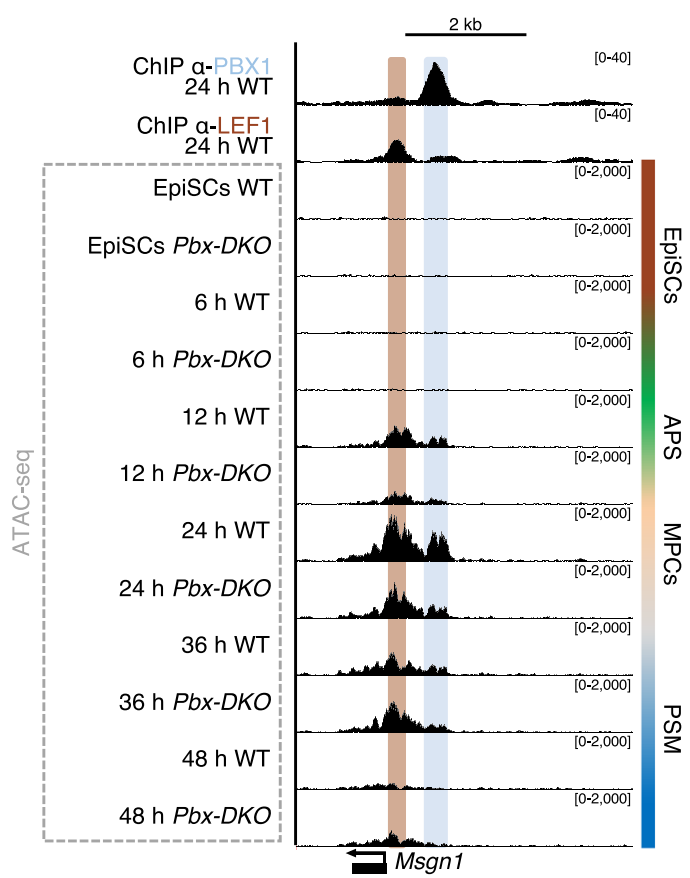

Project License 2017-15-0201-01255 issued by the Danish Regulatory Authority.

Generation of Pbx-DKO lines using the CRISPR/Cas9 system. EpiSC lines derived from E6.5 mouse embryos (129S6/SvEvTac background) were a kind gift from Dr. M.M. Rothová (The Novo Nordisk Foundation Center for Stem Cell Biology,
University of Copenhagen, Copenhagen, Denmark). The lines were derived in EpiSC medium as previously described ${ }^{56}$. $P b x-D K O$ lines were established via sequential CRISPR/Cas9-mediated gene targeting. First, $\mathrm{Pb} \times 2-\mathrm{KO}$ lines were generated by inserting a floxed CMV-hygromycin-Poly (A) cassette into Exon 1 of $P b x 2$ to disrupt $P b x 2$ gene expression, employing the CRISPR Nickase system ${ }^{57}$. Guide RNAs (gRNAs) were designed using the CRISPR/Cas9 design tool (crispr.mit.edu) and cloned into pX335 (a gift from Prof. F. Zhang; Addgene, 42335). Cells were co-transfected with a pair of 
Fig. 6 Sequential recruitment of PBX/HOX-1 complexes to Msgn1 promoter changes the chromatin landscape and activates transcription. a In situ hybridisation shows loss of Msgn1 expression in E8.5 Pbx-com tailbuds. Scale bar: $100 \mu \mathrm{m}$. b Relative Msgn1 mRNA expression measured by RT-qPCR, revealing dynamic expression during PSM differentiation and strong downregulation in Pbx-DKO cells (yellow). c UCSC genome browser snapshot of the murine Msgn1 locus showing conservation across vertebrates. PBX1 (light blue) and LEF1 (brown) are recruited to Msgn1 promoter at 12-24 h. LEF1 binding is lost in Pbx-DKO at any time-points. $\mathbf{d}$ Pseudotime kinetics of lineage markers along PSM differentiation. Prep1 is expressed before PS induction (marked by $T$-Bra), while Pbx1, Meis2, Hox-1 are transcribed within the same time-window of Msgn1. e Dot plot analysis of scRNA-seq data of paraxial mesodermrelated clusters showing enriched expression of Pbx, Meis2, Hox-1 in MPCs at $24 \mathrm{~h}$. $\mathbf{f}$ ChIP-qPCR analyses of TALE proteins at indicated time-points uncover the peak of PREP1 binding at $12 \mathrm{~h}$ and PBX1/MEIS2 at $24 \mathrm{~h} . \mathbf{g}$ Top: schematics of Msgn1 locus and formation of the PBX/MEIS2/HOX-1 complex. Bottom: Msgn1-P2 oligonucleotide sequence (50 bp in the middle of PBX1 ChIP-seq peak) containing WT (upper case) and mutated (lower case) PBX/HOX-1binding site (red). $\mathbf{h}$ EMSA with in vitro-translated TALE proteins and Msgn1-P2 oligonucleotide. The composition of each binding reaction is indicated. A ternary complex (TC) is formed only when PBX, MEIS2 and HOXA1 are mixed together (lines 4-6). TC is co-migrating with a complex present in MPCs nuclear extracts (line 8). The oligonucleotide with single-base substitutions in the PBX/HOX-binding site (Msgn1-P2-M) abrogates all complex formation (lines 12-14). LYS, reticulocyte lysate (lines 7 and 15). i ATAC-seq of Msgn1 locus in WT and Pbx-DKO cells along differentiation reveals specific opening of PBX1 and LEF1-binding sites. ATAC-seq signals in WT mimic Msgn1 transcriptional profile, with maximum accessibility and expression at $24 \mathrm{~h}$. In Pbx-DKO cells, accessibility to Msgn1 is reduced in the LEF1-binding and the PBX/HOX-1-binding regions. Threshold of vertical viewing range of data based on RPKM values is indicated in $\mathbf{c}$ and $\mathbf{i}$. Data are mean \pm s.e.m. $(n=2$ biological replicates) in $\mathbf{b}$ and $\mathbf{f}$.

gRNA-pX335 plasmids (gRNA 1: GTCCAGGCTCTGGTCCGTGA; gRNA 2: CCAA GTGAGTGCCCCCACCC; $100 \mathrm{ng}$ each vector) and $1 \mu \mathrm{g}$ linearised targeting vector using Lipofectamine 2000 (Thermo Fisher Scientific, 11668030), seeded at low density in EpiSC medium in $100 \mathrm{~mm}$ tissue-culture dishes (Corning, 430293) pre-coated with $15 \mu \mathrm{g} / \mathrm{ml}$ human Fibronectin, and selected for hygromycin resistance for 8-10 days. Single colonies were screened by diagnostic PCR, Southern blot and western blot analyses. To reduce side effects caused by CRISPR/Cas9 off-targets, we subsequently employed the High-fidelity CRISPR/Cas9 system (Cas9-HF1) ${ }^{58}$ to target Exon1 of Pbx1 in Pbx2-KO EpiSC lines. The gRNA used (GGCCATCCCGACCCCAGCGT) was cloned into a modified version of pX458 (a gift from Prof. F. Zhang; Addgene, 48138) containing the sequence encoding for Cas9-HF1 (a kind gift from Dr. F.V. Roske (Novo Nordisk, Denmark)). Cells were transfected with 300 ng gRNA-pX458 and after $48 \mathrm{~h}$ GFPpos cells were re-seeded at low density in EpiSC medium in Fibronectin-coated $100 \mathrm{~mm}$ tissue-culture dishes. After 6-8 days, single colonies were screened by diagnostic PCR and selected $P b x$-DKO lines were expanded and validated by Western blot.

Generation of Pbx-DKO lines by CRE-mediated recombination. EpiSC lines were derived in EpiSC medium as previously described ${ }^{56}$ by Dr. J. Martin Gonzalez (Core Facility for Transgenic Mice, Faculty of Health and Medical Sciences, University of Copenhagen, Copenhagen, Denmark) from mixed background $P b x 1^{f l / f l}: \mathrm{Pbx}^{-I}$ mouse embryos. Additionally, $Y f p$ (yellow fluorescent protein) cDNA was inserted at the Rosa26 locus preceded by a loxP-flanked stop sequence to generate CRE recombination reporter lines. After isolation, cell lines were cultured as described above for a few passages, transfected with $200 \mathrm{ng}$ pCAG-Cre (a gift from Prof. C. Cepko; Addgene, 13775) using Lipofectamine 2000 and re-seeded at low density in EpiSC medium in $100 \mathrm{~mm}$ tissue-culture dishes pre-coated with $15 \mu \mathrm{g} / \mathrm{ml}$ human Fibronectin. After $6-8$ days, single YFPpos colonies were picked and screened by PCR. The selected lines were expanded and their genotype was further validated by western blot.

Generation of pMsgn1-mut lines using the CRISPR/Cas9 system. To generate the pMsgn 1-mut lines, CRISPR/Cas9-mediated homology recombination was performed on 129 EpiSCs using a single-stranded DNA (ssDNA) donor template carrying four base substitutions that abrogate the recruitment of PBX to the Msgn 1 promoter, as assessed by EMSA (Fig. $6 \mathrm{~g}-\mathrm{h}$ ). The gRNA designed for targeting the Msgn1 regulatory region (shown in Supplementary Fig. 8a) was cloned into pX459 (a gift from Prof. F. Zhang; Addgene, 62988). Cells were co-transfected with $200 \mathrm{ng}$ gRNA-pX459 and 500 pM ssDNA donor template using Lipofectamine 2000, reseeded at low density in EpiSC medium in $100 \mathrm{~mm}$ tissue-culture dishes pre-coated with $15 \mu \mathrm{g} / \mathrm{ml}$ human Fibronectin and selected for neomycin resistance. Single colonies were screened by diagnostic PCR and restriction enzyme analysis. The selected lines were expanded and the gene editing was further validated by sequencing.

EpiSC differentiation to PSM and derivatives. All differentiation was conducted in serum-free, feeder-free and monolayer conditions in chemically defined N2B27based media. Culture conditions followed a modified protocol from Loh et al. ${ }^{59}$. EpiSCs were seeded at $\sim 6 \times 10^{3}$ cells $/ \mathrm{cm}^{2}$ in six-well cell-culture plates pre-coated with $15 \mu \mathrm{g} / \mathrm{ml}$ human Fibronectin, and cultured in EpiSC medium for $24 \mathrm{~h}$. EpiSCs were first differentiated towards APS using $30 \mathrm{ng} / \mathrm{ml}$ Activin A, $4 \mu \mathrm{M}$ CHIR-99021, $20 \mathrm{ng} / \mathrm{ml} \mathrm{bFGF}$ and $100 \mathrm{nM}$ PIK-90 (APS medium) for $12 \mathrm{~h}$, and then to PSM using $1 \mu \mathrm{M}$ A-83-01, $3 \mu \mathrm{M}$ CHIR-99021, $250 \mathrm{nM}$ LDN193189 and $20 \mathrm{ng} / \mathrm{ml}$ bFGF (PSM medium) for $36 \mathrm{~h}$. For PSM derivatives, cells were kept in PSM medium for additional $24 \mathrm{~h}$, and then differentiated towards ventral somites/sclerotome using $5 \mathrm{nM}$ SAG $21 \mathrm{k}$ and $1 \mu \mathrm{M}$ C59 (sclerotome medium) or dorsal somites/dermomyotome using $3 \mu \mathrm{M}$ CHIR-99021 and $150 \mathrm{nM}$ GDC-0449 (dermomyotome medium) until day 5. A complete list of cytokines and signalling pathway inhibitors used for cell maintenance and differentiation is provided in Supplementary Table 1.

Lentivirus-mediated exogenous expression of Msgn1. The murine Msgn1-coding sequence was amplified from cDNA, subcloned into the VIRSP lentiviral vector between the restriction sites NheI and XhoI and verified by sequencing. In order to produce the lentivirus carrying the VIRSP-Msgn1 vector, HEK293T cells were seeded at $\sim 9 \times 10^{4} \mathrm{cells} / \mathrm{cm}^{2}$ in $100 \mathrm{~mm}$ tissue-culture dishes in Dulbecco's Modified Eagle's Medium (DMEM, Thermo Fisher Scientific, 10566016) + 10\% (v/v) Fetal Bovine Serum (FBS, Gibco, 10270-106). The following day, the medium was changed to $\mathrm{N} 2 \mathrm{~B} 27,1 \mathrm{~h}$ prior to transfection. Cells were transfected with $15 \mu \mathrm{g}$ VIRSP-Msgn1, $10 \mu \mathrm{g}$ psPAX2 (a gift from Prof. D. Trono; Addgene, 12260), $5 \mu \mathrm{g} \mathrm{pCMV-VSV-G} \mathrm{(a} \mathrm{gift} \mathrm{from}$ Prof. R.A. Weinberg; Addgene, 8454) and $90 \mu \mathrm{l} \mathrm{PEI} \mathrm{(1} \mathrm{mg/ml,} \mathrm{Sigma} \mathrm{Aldrich,} \mathrm{765090).}$ The supernatant containing the lentivirus was collected after $72 \mathrm{~h}$ and concentrated using ultracentrifugation filter units (Merck Millipore, UFC910008) as per manufacturer's instructions. The concentrated supernatant was stored at $-80^{\circ} \mathrm{C}$. To perform the rescue experiment, WT and Pbx-DKO 129 EpiSCs were seeded in six-well cellculture plates pre-coated with $15 \mu \mathrm{g} / \mathrm{ml}$ human Fibronectin. In all, $3.5 \mu \mathrm{l}$ of lentivirus were added $4 \mathrm{~h}$ after seeding, and then differentiation to PSM was carried out as described above. Total RNA was extracted from samples harvested after $48 \mathrm{~h}$ of differentiation and the rescue efficiency was assessed by RT-qPCR as compared to WT EpiSCs with and without added virus, as well as to $P b x-D K O$ cells without added virus.

Immunofluorescence staining of adherent cells. EpiSCs were seeded at $\sim 4 \times 10^{3} \mathrm{cells} / \mathrm{cm}^{2}$ in EpiSC medium in eight-well microscopy slides (Ibidi, 80826) pre-coated with $15 \mu \mathrm{g} / \mathrm{ml}$ human Fibronectin, and differentiated to PSM as described above. At desired time-points, cells were briefly washed twice in PBS (Sigma, D8537) and fixed in 4\% (w/v) paraformaldehyde diluted in PBS from $16 \%$ methanol-free formaldehyde stock (Pierce Biotechnologies, 28906) for $15 \mathrm{~min}$ at RT. Fixed cells were then permeabilised in PBS containing $0.1 \%$ (v/v) Triton X-100 (PBST) for $10 \mathrm{~min}$ at RT and blocked in 3\% (v/v) Donkey serum in PBST containing 1\% (w/v) BSA (blocking solution) for $15 \mathrm{~min}$ at RT. Primary and secondary antibody incubations were done in blocking solution overnight at $4{ }^{\circ} \mathrm{C}$ and for $2 \mathrm{~h}$ at RT, respectively. Antibody incubations were followed by three quick washes in PBST followed by two washes in PBST for $15 \mathrm{~min}$ at RT. After the final incubation, cells were stored in PBST containing $1 \mu \mathrm{g} / \mathrm{ml}$ DAPI at $4^{\circ} \mathrm{C}$, packed in Parafilm to prevent evaporation. Cells were always kept in the dark after incubation with secondary antibodies. Confocal imaging was performed using a Leica TCS SP8 microscope and images were processed using the Imaris ${ }^{\mathrm{TM}}$ software (v9.3.0) with default settings. The antibodies used are listed in Supplementary Table 2.

Imaging of embryo morphology. Embryos were dissected in cold PBS, fixed in 4\% (w/v) paraformaldehyde for $30 \mathrm{~min}$ at RT and washed twice in cold PBS. Images were acquired using a Zeiss AxioZoom.V16 microscope at the Core Facility for Integrated Microscopy (University of Copenhagen, Copenhagen, Denmark).

Immunofluorescence staining of embryos. Embryos were dissected in cold PBS and fixed in $4 \%(\mathrm{w} / \mathrm{v})$ paraformaldehyde for $30 \mathrm{~min}$ at RT. Fixed embryos were rinsed in cold PBS containing $3 \mathrm{mg} / \mathrm{ml}$ PVP (PBS/PVP), permeabilised in PBS/ PVP $+1 \%(\mathrm{v} / \mathrm{v})$ Triton X-100 for $10 \mathrm{~min}$ at RT and blocked in CAS-Block (Thermo Fisher Scientific, 008120 ) overnight at $4{ }^{\circ} \mathrm{C}$. Incubation with the primary antibodies listed in Supplementary Table 3 was carried out in CAS-Block for $48 \mathrm{~h}$ at $4^{\circ} \mathrm{C}$. Embryos were quickly washed three times with CAS-Block at RT and then overnight at $4{ }^{\circ} \mathrm{C}$. Incubation with secondary antibodies was done in CAS-Block for $24 \mathrm{~h}$ at $4{ }^{\circ} \mathrm{C}$. Embryos were quickly washed three times with CAS-Block at RT and 
a

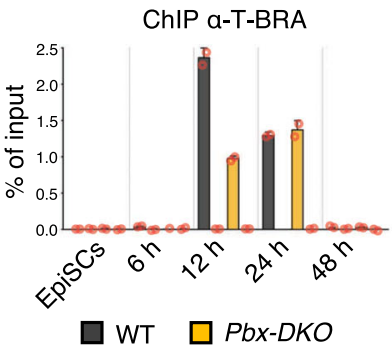

d

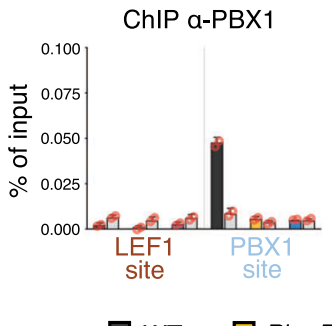

WT

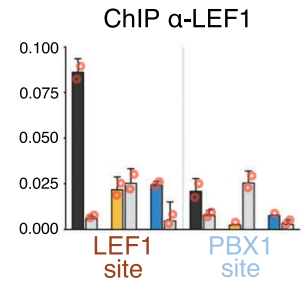

pMsgn1-mut

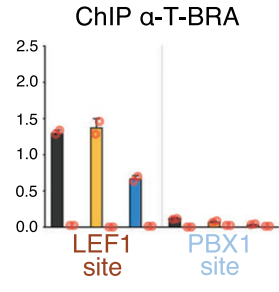

O ChIP a-lgG b

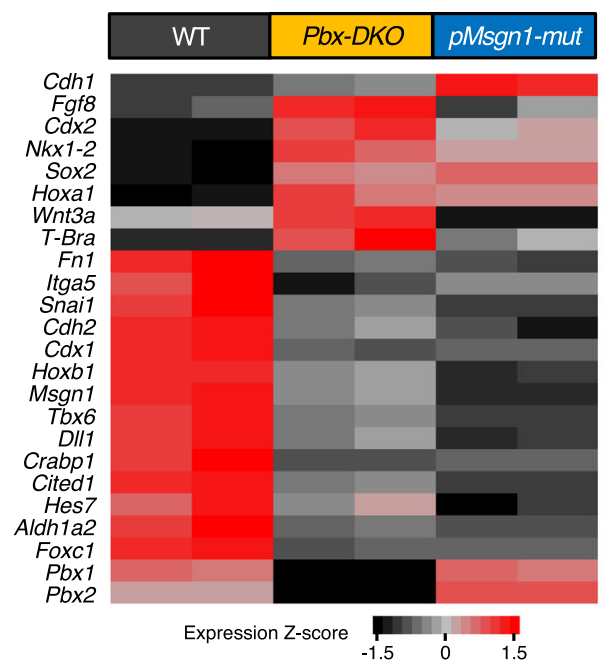

C

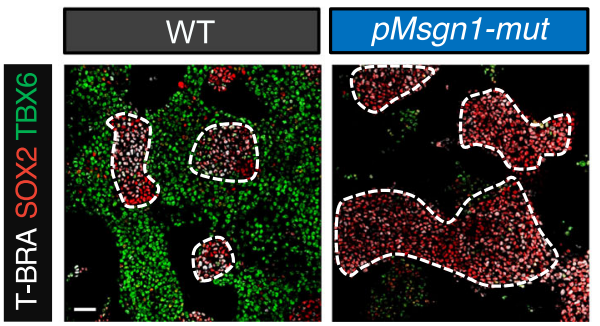

e

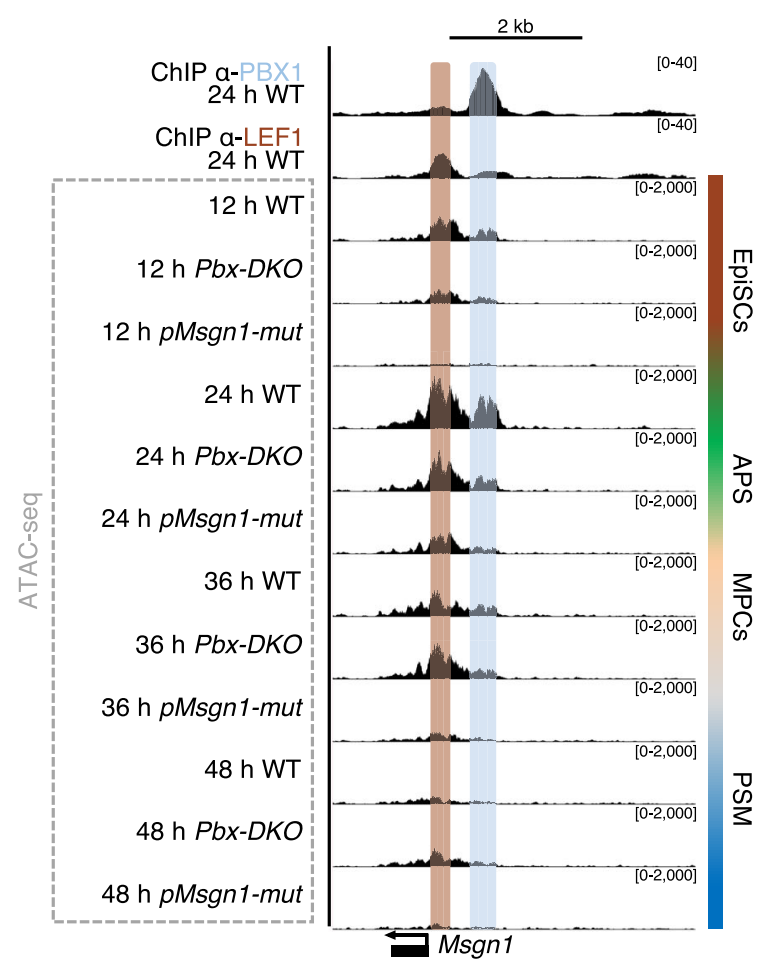

Fig. 7 Combined pioneering activity of T-BRA and TALE proteins remodels the chromatin, making it accessible at Msgn1 regulatory regions, thereby directing WNT transcriptional response. a ChIP-qPCR analysis showing specific recruitment of T-BRA to the LEF1-binding site of the Msgn1 regulatory region at 12 and $24 \mathrm{~h}$. T-BRA binding is maintained in $P b x$-DKO cells (yellow). b Heatmap displaying relative expression of selected differentially regulated genes in WT (black), Pbx-DKO (yellow) and pMsgn1-mut cells (blue) at $24 \mathrm{~h}$ (RNA-seq, $P \leq 0.05$, fold-change $\geq 1.5$ ). pMsgn1-mut allele carries point mutations in the PBX/HOX-1-binding site. Representative PSM and NMP markers are indicated. $n=2$ biological replicates. Significance was assessed by DESeq2 on the basis of two-sided Wald test with Benjamini-Hochberg adjusted P-values. c Representative immunofluorescence staining for T-BRA (white), SOX2 (red) and TBX6 (green) at $48 \mathrm{~h}$ of differentiation reveals expansion of NMPs (T-BRA ${ }^{\text {pos; SOX2 }}{ }^{\text {pos }}$, dashed white lines) and reduction of PSM (TBX6Pos) in pMsgn1-mut cells. Scale bar: $50 \mu \mathrm{m}$. d ChIP-qPCR analysis on the Msgn1 promoter displays PBX1 recruitment at the PBX/HOX-1-binding site in WT (black), but not in Pbx-DKO (yellow) nor in pMsgn1-mut cells (blue). Similarly, ChIP-qPCR analyses with LEF1 and T-BRA antibodies highlight specific binding of LEF1 and T-BRA to the LEF1-binding region in WT cells. While T-BRA is recruited to the Msgn1 promoter in Pbx-DKO and pMsgn1-mut cells, LEF1 binding is lost in both. Data are mean \pm s.e.m. ( $n=2$ biological replicates) in a and d. e Chromatin accessibility analysis (ATAC-seq) of the Msgn1 locus in WT, Pbx-DKO and pMsgn1-mut cells along PSM differentiation displays specific open regions overlapping with the PBX (light blue) and LEF1-binding sites (brown) in WT cells at 12 and $24 \mathrm{~h}$. In Pbx-DKO cells, accessibility is strongly reduced at the PBX/HOX-1-binding region. Additionally, accessibility of the Msgn1 locus is severely impaired in both LEF1 and PBX/HOX-1-binding regions in pMsgn1-mut cells, confirming the role of the PBX complexes as modulators of chromatin accessibility on WNT-responsive elements. Threshold of vertical viewing range of data based on RPKM values is specified.

then overnight at $4{ }^{\circ} \mathrm{C}$. Following incubation with CAS-Block containing $1 \mu \mathrm{g} / \mathrm{ml}$ DAPI for $1 \mathrm{~h}$ at RT, embryos were washed three times with PBS $+0.5 \%$ (v/v) Triton X-100 for $15 \mathrm{~min}$ at RT and dehydrated in ascending series to $100 \%$ methanol. To reduce light scattering, optical clearing was performed by submerging the stained embryos in a 1:2 (v/v) solution of benzyl alcohol:benzyl benzoate (BABB). Images were acquired using a Leica TCS SP8 confocal microscope. Image processing and $3 \mathrm{D}$ reconstruction were performed using the Imaris ${ }^{\mathrm{TM}}$ software (v9.3.0). In Figs. 2c, d and $3 \mathrm{i}$ and Supplementary Fig. 2b, only cells expressing high levels of either T-BRA, SOX2 or TBX6 were included in the scoring.
In situ hybridisation. Embryos were dissected in cold PBS and fixed in 4\% (w/v) paraformaldehyde for $2 \mathrm{~h}$ at RT, washed twice in cold PBS with $0.1 \%$ Tween- 20 and dehydrated in ascending series to $100 \%$ methanol. At least three embryos of the same genotype were used. Whole-mount in situ hybridisation was performed following the protocol used in Prof. E. De Robertis' laboratory (http://www.hhmi.ucla.edu/ derobertis/protocol_page/Pdfs/Mouse\%20protocols/Whole\%20mount\%20in\%20situ $\% 20$ hybridization\%20on\%20mouse\%20embryos.pdf). The antisense riboprobes to $M s g n 1^{60}$ and $W n t 3 a^{61}$ were a kind gift from Prof. A.K. Hadjantonakis (Memorial Sloan Kettering Cancer Center, New York, United States). 
a
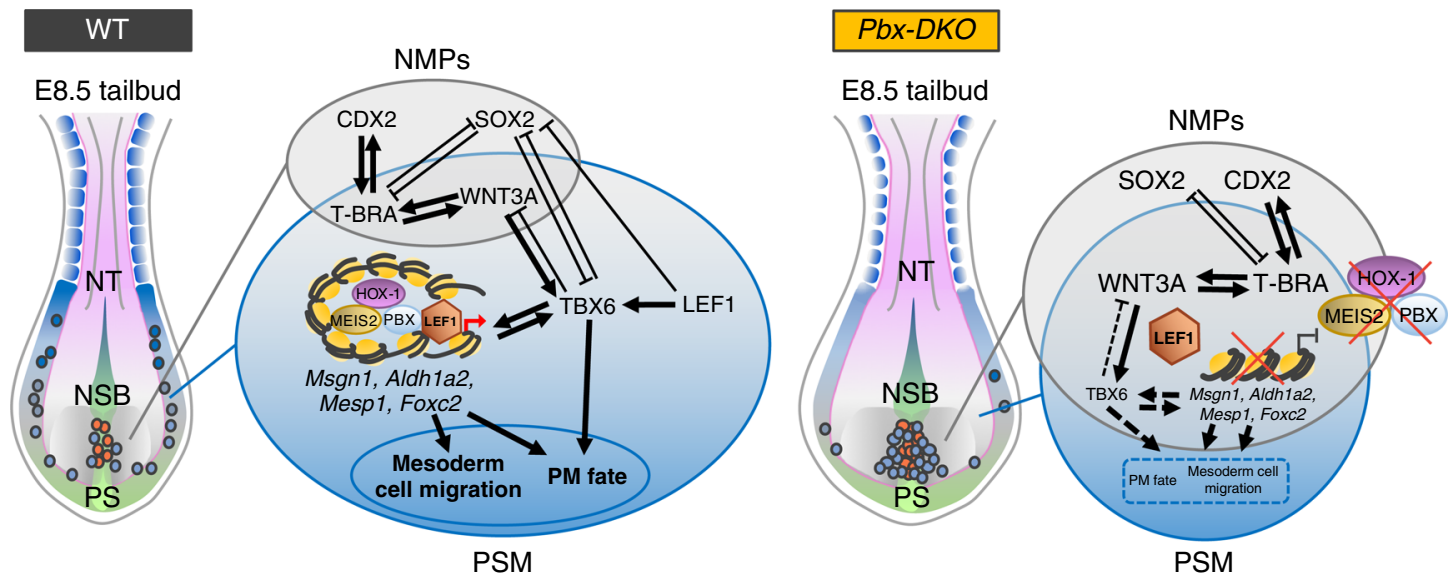

b

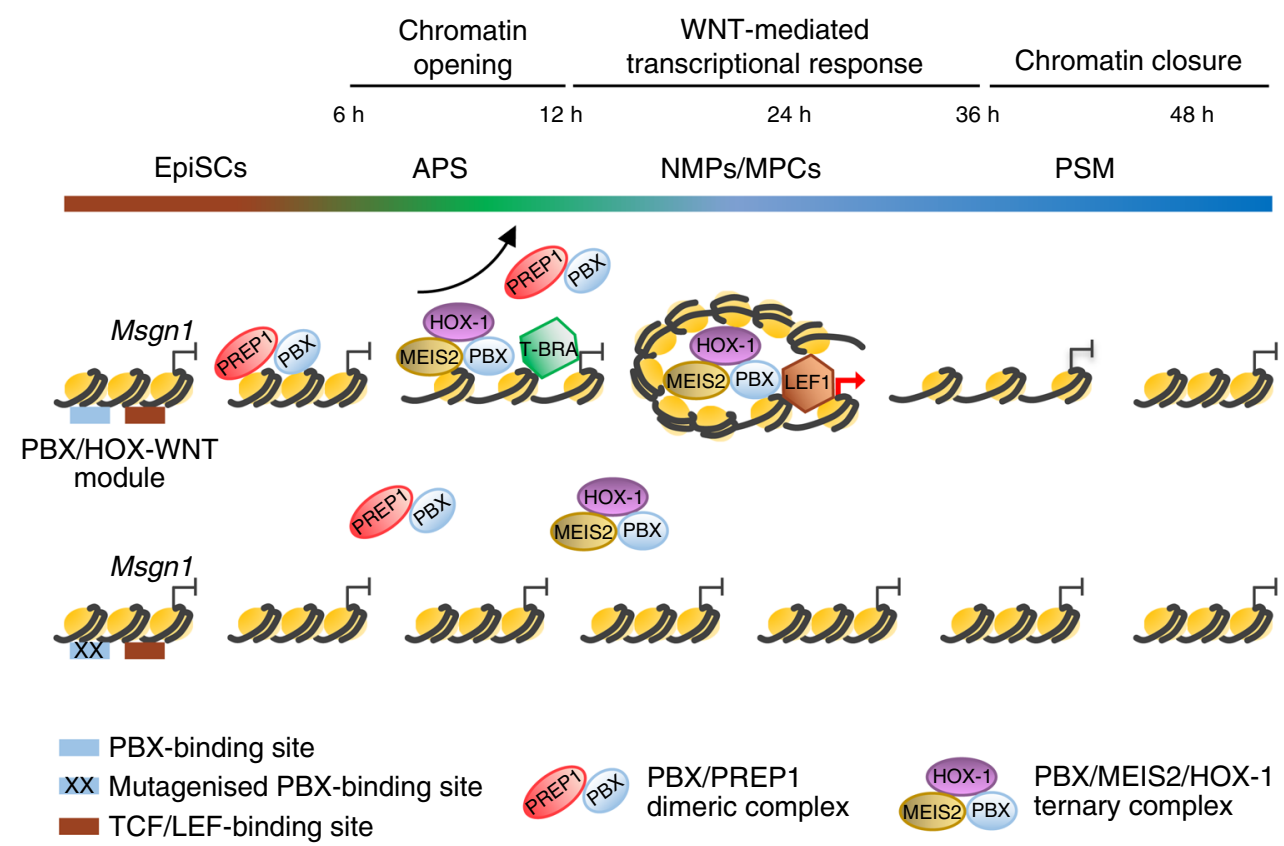

Fig. 8 TALE complexes promote WNT-mediated transcriptional response in paraxial mesoderm. a Schematics of the molecular networks at play in the E8.5 embryonic tailbud. Left: cartoon showing NMPs (red dots) transitioning to MPCs (light blue dots) and migrating to the PSM (blue) in WT embryos. GRNs governing the balance between NMP expansion in the CLE (grey) and PSM differentiation (blue) are illustrated. The TALE complexes and LEF1 bind cooperatively to the regulatory regions of PM genes, like Msgn1, Aldh1a2, Mesp1 and Foxc2, promoting their expression. The activation of the PM programme ultimately controls migration of MPCs from the progenitor zone to the PSM and the acquisition of PM fate. Right: PBX loss (yellow) results in reduced mobility of the MPCs and accumulation of NMPs-MPCs in the progenitor zone, leading to enlarged tailbuds and reduced PSM formation. Failure of TALE complex assembly on the regulatory regions of PM genes impairs LEF1 recruitment and activation of the GRN promoting PSM formation. In contrast, the molecular circuits sustaining NMP expansion are maintained by positive feedback loops. Of note, the observed PBX-LEF1 binding to the N1 repressive element of the Sox2 regulatory region suggests that PBX and LEF1 cooperative activity could repress Sox2 expression (Fig. 5e) and NMP maintenance. Thus, PBX proteins are required for the transition of NMPs to PSM, playing essential and previously unappreciated roles in the early stages of somitogenesis. CLE caudal lateral epiblast, NSB node-streak border, PS primitive streak, PSM pre-somitic mesoderm, NT neural tube. b Model of the transcriptional activation of Msgn1. Msgn1 regulatory region becomes accessible at $12 \mathrm{~h}$ following the collaborative pioneering activity of T-BRA and the TALE complexes. Low-affinity PBX/PREP1 dimers are recruited first and could serve as HOX-1 attractors, or assist the loading of the high-affinity PBX/MEIS2/HOX-1 ternary complex, whose cooperative binding displaces nucleosomes and generates the suitable chromatin context for LEF1 recruitment. Single-base substitutions at the $\mathrm{PBX} / \mathrm{HOX}-1$-binding site abrogate accessibility of both PBX/HOX-1 and LEF1-binding regions, emphasising the role of TALE complexes as modulators of chromatin accessibility on WNT-responsive elements.

Time-lapse videos of PSM differentiation. EpiSCs were seeded at $\sim 6 \times 10^{3}$ cells $/ \mathrm{cm}^{2}$ in six-well cell-culture plates pre-coated with $15 \mu \mathrm{g} / \mathrm{ml}$ human Fibronectin, and differentiated to PSM as described above. Differentiation was carried out in the IncuCyte S3 Live-Cell Analysis System (Essen Bioscience) at the High-Throughput Cell-based Screens Facility (Biotech Research \& Innovation Centre, University of Copenhagen,
Copenhagen, Denmark), and images were acquired every h for 3 days. Time-lapse movies with all acquired pictures were further recreated using iMovie (version 10.1.14)

Western blot. In all, $80 \%$ confluent cells were washed once with PBS and then lysed directly on the plate by addition of 1x Laemmli sample buffer ( $2 \%(w / v)$ SDS, 
$10 \%(\mathrm{v} / \mathrm{v})$ glycerol and $120 \mathrm{mM}$ Tris- $\mathrm{HCl} \mathrm{pH} 6.8)$ to the culture dish $\left(20 \mu \mathrm{l} / \mathrm{cm}^{2}\right)$, followed by extensive scraping using a pipette tip. Samples were vortexed, centrifuged briefly and then stored at $-20^{\circ} \mathrm{C}$ until use. Before loading, samples were boiled for $5 \mathrm{~min}$ at $80^{\circ} \mathrm{C}$, sonicated on ice for $10 \mathrm{~s}$ at $20 \%$ power using a Branson Digital Sonifier Model S-450D (Marshall Scientific), spun for $10 \mathrm{~min}$ at $14,000 \times g$ to clear the lysates and then stored at $-20^{\circ} \mathrm{C}$ until use. Protein concentration was estimated using NanoDrop (Thermo Fisher Scientific) A280 measurements and equal amounts of proteins were diluted in $60 \mu \mathrm{l}$ of $1 \mathrm{x}$ Laemmli sample buffer per sample. After addition of $6 \mu \mathrm{l}$ of $1 \mathrm{M}$ DTT containing $0.01 \%(\mathrm{w} / \mathrm{v})$ bromophenol blue, $50 \mu \mathrm{l}$ of sample were loaded per lane on NuPAGE Novex $4-12 \%$ Bis-Tris Protein Gels (Thermo Fisher Scientific, NP0321BOX). Electrophoresis was performed in 1x NuPAGE MES SDS running buffer (Thermo Fisher Scientific, $\mathrm{NP} 0002)$ at $150 \mathrm{~V}$ for $1 \mathrm{~h}$. Resolved proteins were transferred to Amersham Protran Nitrocellulose Blotting membrane $0.45 \mu \mathrm{m}$ (GE Healthcare, 10600003) at $400 \mathrm{~mA}$ for $90 \mathrm{~min}$ on ice in 1x NuPAGE transfer buffer (Thermo Fisher Scientific, NP0006). After a quick wash in PBS containing $0.1 \%(\mathrm{v} / \mathrm{v})$ Tween-20 (PBST), membranes were blocked for $1 \mathrm{~h}$ at RT in PBST containing 10\% Skim milk powder. All subsequent antibody incubations were performed in PBST containing 5\% (w/v) BSA and followed by three washes in PBST for $10 \mathrm{~min}$. The following primary antibodies were used: polyclonal Rabbit anti-PBX1 (CST, 4342, 1:1,000), polyclonal Rabbit anti-PBX2 (Santa Cruz Biotechnology, sc-890, 1:3,000) and monoclonal Mouse anti-H3 (abcam, ab10799, 1:30,000). The secondary antibodies used include: Donkey anti-Rabbit HRP (Thermo Fisher Scientific, A16023, 1:10,000) and Donkey anti-Mouse HRP (Thermo Fisher Scientific, A16011, 1:10,000). For chemiluminescence detection, the membrane was incubated with Amersham ECL Prime Western Blotting Detection reagent (GE Healthcare, RPN2236) and imaged on a ChemiDoc MP (Bio-Rad). For reprobing with different primary antibodies, the membrane was soaked in Restore PLUS Western Blot Stripping Buffer (Thermo Fisher Scientific, 46430) two times for $10 \mathrm{~min}$ at RT to inactivate horseradish peroxidase (HRP). To ensure comparable densitometry results, gel electrophoresis, protein transfer and antibody staining were performed in parallel for all samples used to generate each western blot figure panel.

Electrophoretic Mobility Shift Assay. EMSAs were performed as described ${ }^{62}$, using in vitro-translated proteins or nuclear extracts purified from cells. Sequences of the oligonucleotides spanning the $\mathrm{PBX} / \mathrm{HOX}-1$-binding site on the Msgn 1 promoter used in EMSA are described in Supplementary Fig. 7a. EMSAs with in vitro-translated proteins were performed using $5 \mu \mathrm{g}$ of nuclear extract or $2 \mu \mathrm{l}$ of reticulocyte lysate containing the desired combination of proteins mixed with $18 \mu \mathrm{l}$ of PPH binding buffer (10 mM Tris-Cl pH 7.5, $75 \mathrm{mM} \mathrm{NaCl}, 1 \mathrm{mM}$ EDTA, $6 \%$ (v/v) glycerol, $3 \mathrm{mM}$ spermidine, $1 \mathrm{mM}$ DTT, $0.5 \mathrm{mM}$ PMSF, $1 \mu \mathrm{g}$ poly (dIdC), 30,000 c.p.m ${ }^{32} \mathrm{P}$-labelled oligonucleotide) to a total volume of $20 \mu \mathrm{l}$. After $30 \mathrm{~min}$ of incubation on ice the reactions were separated by $5 \%$ acrylamide gel in $0.5 \mathrm{x}$ TBE. For the competition assays 50, 100 or 200-molar excess of unlabelled competitor oligonucleotide were added to the binding reaction $10 \mathrm{~min}$ before the labelled probe. Acrylamide gels were pre-run at $300 \mathrm{~V}$ for $90 \mathrm{~min}$. Samples were separated on gel at $150 \mathrm{~V}$ for $2 \mathrm{~h}$. Gels were dried on gel dryer, exposed overnight and imaged on a Typhoon 9400 imager (GE Healthcare). Based on the known molecular weight of the in vitro-translated proteins, the expected sizes of dimeric and ternary complexes are as follows: Fig. $6 \mathrm{~h}$ : PBX1A/MEIS2 $=\sim 114 \mathrm{kDa}$, PBX1B/MEIS2 $=\sim 104 \mathrm{kDa}$, PBX2/MEIS2 $=\sim 114 \mathrm{kDa}$, PBX1A/MEIS2/HOXA1 $=\sim 149 \mathrm{kDa}, \mathrm{PBX} 1 \mathrm{~B} / \mathrm{MEIS} 2 / \mathrm{HOXA} 1=\sim 139 \mathrm{kDa}, \mathrm{PBX} 2 /$ MEIS2/HOXA1 $=\sim 149 \mathrm{kDa}$; Supplementary Fig. $7 \mathrm{c}$ : PBX1A/MEIS2 $=\sim 114 \mathrm{kDa}$, PBX1B/MEIS2 $=\sim 104 \mathrm{kDa}$, PBX2/MEIS2 $=\sim 114 \mathrm{kDa}$, PBX3/MEIS2 $=\sim 114 \mathrm{kDa}$, PBX1A/MEIS2/HOXA1 $=\sim 149 \mathrm{kDa}$, PBX1B/MEIS2 $/ \mathrm{HOXA} 1=\sim 139 \mathrm{kDa}, \mathrm{PBX} 2 /$ MEIS2/HOXA1 $=\sim 149 \mathrm{kDa}$, PBX3/MEIS2/HOXA1 $=\sim 149 \mathrm{kDa}$, PBX1A/MEIS2 $/$ HOXB1 $=\sim 146 \mathrm{kDa}, \mathrm{PBX} 1 \mathrm{~B} / \mathrm{MEIS} 2 / \mathrm{HOXB} 1=\sim 136 \mathrm{kDa}$, PBX2/MEIS2 $/ \mathrm{HOXB1}=$ $\sim 146 \mathrm{kDa}$, PBX3/MEIS2/HOXB1 $=\sim 146 \mathrm{kDa}$; Supplementary Fig. 7d: PBX1A/PRE $\mathrm{P} 1=\sim 114 \mathrm{kDa}, \mathrm{PBX} 1 \mathrm{~B} / \mathrm{PREP} 1=\sim 104 \mathrm{kDa}, \mathrm{PBX} / \mathrm{PREP} 1=\sim 114 \mathrm{kDa}, \mathrm{PBX} 3 / \mathrm{PRE}$ $\mathrm{P} 1=\sim 114 \mathrm{kDa}$, PBX1A/MEIS2 $=\sim 114 \mathrm{kDa}$, PBX1B/MEIS2 $=\sim 104 \mathrm{kDa}$, PBX2/MEI $\mathrm{S} 2=\sim 114 \mathrm{kDa}$, PBX3/MEIS2 $=\sim 114 \mathrm{kDa}$; Supplementary Fig. 7e: PBX1A/PREP1 = $\sim 114 \mathrm{kDa}$, PBX1A/PREP1/HOXA1 $=\sim 149 \mathrm{kDa}$, PBX1A/MEIS2 $=\sim 114 \mathrm{kDa}$, PBX1 A/MEIS2/HOXA1 $=\sim 149 \mathrm{kDa}$; Supplementary Fig. 7f: PBX3/PREP1 $=\sim 114 \mathrm{kDa}$, PBX1A/MEIS2/HOXB1 = 146 kDa; and Supplementary Fig. 7g: PBX1A/PREP1 = $\sim 114 \mathrm{kDa}, \mathrm{PBX} 1 \mathrm{~A} / \mathrm{MEIS} 2 / \mathrm{HOXB} 1=\sim 146 \mathrm{kDa}$

Oligonucleotides preparation. Oligonucleotides were resuspended in TE $\mathrm{pH} 8.0$. Double-stranded oligonucleotides were prepared by mixing equal molar ratios of single-strand oligonucleotides. The mixture was heated at $90^{\circ} \mathrm{C}$ for $10 \mathrm{~min}$ and slowly cooled at RT. Perfect oligo annealing was checked by electrophoresis on 15\% acrylamide gel. For oligos longer than $40 \mathrm{bp}$, double-strand oligonucleotides purification was performed using preparative acrylamide $15 \%$ in $0.5 \mathrm{x}$ TBE. The oligonucleotides were eluted from the gel in $500 \mu \mathrm{l}$ TEN $(10 \mathrm{mM}$ Tris, $1 \mu \mathrm{M}$ EDTA, $300 \mathrm{mM} \mathrm{NaCl}, \mathrm{pH}$ 8.0) with overnight shaking. The oligonucleotides were recovered by ethanol precipitation. The pellet was washed, dried and resuspended in $50 \mu \mathrm{l} \mathrm{TE} \mathrm{pH} \mathrm{8.0.}$

Oligonucleotides $\mathbf{5}^{\prime}$ end labelling. In total, 0.5 pmoles of double-stranded oligonucleotides were incubated with $1 \mu \mathrm{l}$ of $10 \mathrm{x}$ PNK buffer 5 Units Polynucleotide Kinase (Roche, 10174645001) and [ $\left.\gamma^{-32} \mathrm{P}\right]$ ATP $3000 \mathrm{Ci} / \mathrm{mMole}$ (PerkinElmer,
BLU502A250UC). The reaction was incubated at $37^{\circ} \mathrm{C}$ for $40 \mathrm{~min}$, diluted to $50 \mu \mathrm{l}$ with TE pH 8.0 and purified on a packed Sepharose G-25 column (Roche, 11273949001).

Nuclear extract preparation. Nuclear extracts (NE) for EMSA were prepared from in vitro-differentiated paraxial mesoderm cells. Cells were resuspended in $200 \mu \mathrm{l}$ lysis buffer (10 mM HEPES pH 7.9, $30 \mathrm{mM} \mathrm{KCl}, 1.5 \mathrm{mM} \mathrm{MgCl}_{2}, 1 \mathrm{mM}$ DTT, supplemented with $1 \mathrm{x}$ Protease inhibitor cocktail (Roche, 11836170001)), kept on ice for $10 \mathrm{~min}$ and supplemented with addition of Triton X-100 to a final concentration of $0.1 \%$. The nuclei were collected by centrifugation at $4{ }^{\circ} \mathrm{C}$. Nuclear extracts were prepared by resuspending the nuclear pellet in $50 \mu \mathrm{l}$ nuclear buffer (20 mM HEPES pH 7.9, 25\% (v/v) glycerol, $0.42 \mathrm{M} \mathrm{NaCl}, 1.5 \mathrm{mM} \mathrm{MgCl}_{2}, 1 \mathrm{mM}$ DTT, supplemented with $1 \mathrm{x}$ Protease inhibitor cocktail). NE preparations were incubated at $4{ }^{\circ} \mathrm{C}$ with rotation for $30 \mathrm{~min}$ and cleared by centrifugation. Finally, the extracts were quantified by NanoDrop, aliquoted and stored at $-80^{\circ} \mathrm{C}$

Cell-free protein synthesis. The cDNAs were transcribed and translated using the couple T7-TNT reticulocytes lysate system (Promega, L1170) according to the manufacturer's instructions. All cDNAs of the proteins used in this study (PBX1A, PBX1B, PBX2, PBX3A, PREP1, PREP2, MEIS2, HOXA1 and HOXB1) were cloned in vectors under the control of T7 promoter. The PBX3A (MC202386) and PREP2 (MC204820) constructs were purchased from OriGene Technologies. All the other plasmids were described in Ferretti et al. ${ }^{62}$.

scRNA-seq procedure. scRNA-seq was performed using the Massively Parallel Single-Cell RNA-sequencing technology (MARS-seq ${ }^{63}$ ). For in vitro experiments, EpiSCs were seeded at $\sim 6 \times 10^{3}$ cells $/ \mathrm{cm}^{2}$ in six-well cell-culture plates pre-coated with $15 \mu \mathrm{g} / \mathrm{ml}$ human Fibronectin, and differentiated to PSM as described above. At desired time-points, cells were dissociated using Accutase (Thermo Fisher Scientific, 00-4555-56) for $3 \mathrm{~min}$ at $37^{\circ} \mathrm{C}$ and counted. 500,000 cells per condition were washed with ice-cold FACS buffer (10\% (v/v) FBS in PBS) and resuspended into $1 \mathrm{ml}$ ice-cold FACS buffer containing $1 \mu \mathrm{g} / \mathrm{ml}$ DAPI. For in vivo experiments, tailbuds were dissected from E8.5/E9.0 embryos and dissociated into $300 \mu$ l warmed Trypsin $0.05 \%(\mathrm{w} / \mathrm{v})$ EDTA for $10 \mathrm{~min}$ at $37^{\circ} \mathrm{C}$. Digestion was stopped by addition of $600 \mu \mathrm{l}$ ice-cold FACS buffer followed by centrifugation at $350 \times g$ for $3 \mathrm{~min}$. Cells were washed with $500 \mu$ ice-cold FACS buffer, centrifuged again at $350 \times g$ for $3 \mathrm{~min}$ and resuspended into $250 \mu \mathrm{l}$ ice-cold FACS buffer containing $1 \mu \mathrm{g} / \mathrm{ml}$ DAPI Singlecells from in vitro-differentiated cells or embryonic tailbuds were sorted into Eppendorf Polypropylene U-shaped 384-well Twin Tec PCR Microplates (Thermo Fisher Scientific, 10573035), containing $2 \mu \mathrm{l}$ of lysis solution $(0.2 \%(\mathrm{v} / \mathrm{v})$ Triton X100) supplemented with $0.4 \mathrm{U} / \mu \mathrm{l}$ RNasin Ribonuclease Inhibitor (Promega, N2515) and $400 \mathrm{nM}$ indexed RT primer from group 1 (1-96 barcodes) or group 2 (97-192 barcodes ${ }^{63}$. Additionally, 71 WT EpiSCs were sorted into each plate, as spike-in control for batch-effect correction. Capture plates were prepared on the Bravo automated liquid handling robot station (Agilent) using 384-filtered tips (Agilent, 19133-142). Index sorting was performed using a FACS Aria III cell sorter (BD Biosciences) at the DanStem Flow Cytometry Platform (University of Copenhagen, Copenhagen, Denmark), sorting live cells as singlets. Immediately after sorting, plates were spun down, snap-frozen on dry ice and stored at $-80^{\circ} \mathrm{C}$ until further processing. Semi-automated library preparation was performed, using 10-12 total cycles of PCR amplification and AMPure XP beads (Beckman Coulter Life Sciences, A63881) for purification ${ }^{63}$. DNA concentration was measured with a Qubit Fluorometer (Thermo Fisher Scientific, Q32854) and fragment size was determined with a Fragment analyzer (Agilent). Libraries were paired-end sequenced on a Next-Seq 500 Sequencer (Illumina) at the DanStem Genomics Platform (University of Copenhagen, Copenhagen, Denmark). Between 1,146 and 1,528 cells were sequenced per lane.

RNA-seq procedure. EpiSCs were seeded at $\sim 6 \times 10^{3}$ cells $/ \mathrm{cm}^{2}$ in six-well cellculture plates pre-coated with $15 \mu \mathrm{g} / \mathrm{ml}$ human Fibronectin, and differentiated to PSM as described above. At desired time-points, cells were lysed and total RNA was extracted using the RNeasy Mini Kit according to the manufacturer's instructions (Qiagen, 74106). RNA concentration was quantified by NanoDrop and quality was verified with a Fragment analyzer. Library preparation was carried out using $0.5 \mu \mathrm{g}$ of RNA with NEBNext Ultra II RNA library Prep Kit (New England BioLabs, E7770S) as per manufacturer's instructions. Libraries were amplified for 4 total PCR cycles and purified with AMPure XP beads. DNA concentration was measured with a Qubit Fluorometer and fragment size was determined with a Fragment analyzer. All samples were sequenced in biological triplicates on a Next-Seq 500 Sequencer (Illumina) at the DanStem Genomics Platform (University of Copenhagen, Copenhagen, Denmark).

ATAC-seq procedure. ATAC-seq was performed on 50,000 cells per replicate as described in Buenrostro et al. ${ }^{64}$ (with modifications based on Corces et al. ${ }^{65}$ ), on EpiSCs and PSM-differentiated cell populations at desired time-points. Libraries were generated using the Ad1_noMX and Ad2.1-2.16 barcoded primers ${ }^{64}$ and amplified for 10 total PCR cycles. Libraries were purified with AMPure XP beads to remove contaminating primer dimers and fragments $>1,000 \mathrm{bp}$. Library quality was assessed using the Fragment analyzer and quantitated by Qubit assay. The libraries were sequenced with $50 \mathrm{bp}$ 
paired-end reads on a Next-Seq 500 Sequencer (Illumina) at the DanStem Genomics Platform (University of Copenhagen, Copenhagen, Denmark).

ChIP-seq procedure. ChIP was performed with a protocol modified from that of Kolasinska-Zwierz et al. ${ }^{66}$. EpiSCs were seeded at $\sim 2 \times 10^{3}$ cells $/ \mathrm{cm}^{2}$ in EpiSC medium in $150 \mathrm{~mm}$ tissue-culture dishes (Corning, 430599) pre-coated with $15 \mu \mathrm{g}$ / $\mathrm{ml}$ human Fibronectin, and differentiated to PSM as described above. At desired time-points, cells were washed with PBS and fixed directly on the plate by the addition of $20 \mathrm{ml}$ of PBS containing 1\% (v/v) Formaldehyde Fixative Ultrapure EM Grade (Rockland, KHF001) for $8-10 \mathrm{~min}$ at RT. Fixation was stopped by $5 \mathrm{~min}$ incubation with $125 \mathrm{mM}$ glycine at RT. After two washes with cold PBS, fixed cells were harvested in lysis buffer $(50 \mathrm{mM}$ Tris- $\mathrm{HCl} \mathrm{pH} 8.1,100 \mathrm{mM} \mathrm{NaCl}, 5 \mathrm{mM}$ EDTA, $0.2 \%(\mathrm{w} / \mathrm{v}) \mathrm{NaN}_{3}, 0.5 \%$ (w/v) SDS, supplemented with 1x Protease inhibitor cocktail and $1 \mathrm{mM}$ PMSF) by extensive scraping using a pipette tip. Following centrifugation at $1,000 \times \mathrm{g}$ for $5 \mathrm{~min}$ at $4{ }^{\circ} \mathrm{C}$, the pellet was resuspended in $1 \mathrm{ml}$ of FA buffer (50 mM HEPES/KOH pH 7.5, $1 \mathrm{mM}$ EDTA, 1\% (v/v) Triton X-100, $0.1 \%$ sodium deoxycholate, $150 \mathrm{mM} \mathrm{NaCl}$, supplemented with $1 \mathrm{x}$ Protease inhibitor cocktail) per $0.5 \mathrm{ml}$ of pellet. Chromatin was disrupted by sonication using a chilled Bioruptor Plus sonicator UCD-300 (Diagenode) for 8-12 pulses (cycles of $30 \mathrm{~s}$ on $/ 45 \mathrm{~s}$ off, high setting at $4^{\circ} \mathrm{C}$ ) to obtain fragments of $200-500 \mathrm{bp}$ in size. The extract was spun for $30 \mathrm{~min}$ at $20,000 \times g$ at $4{ }^{\circ} \mathrm{C}$, and the soluble fraction was aliquoted and stored at $-80^{\circ} \mathrm{C}$ until use. For each IP, $20 \mu \mathrm{g}$ of chromatin were incubated overnight in $1 \mathrm{ml}$ of FA buffer containing 1\% N-lauroylsarkosine sodium salt with the antibodies listed in Supplementary Table $4.10 \%$ of the IP volume of the chromatin was retained as an input reference. After overnight rotation at $4{ }^{\circ} \mathrm{C}$, $30 \mu \mathrm{l}$ of washed and blocked magnetic Protein A (Thermo Fisher Scientific, 10006D) or Protein G Dynabeads (Thermo Fisher Scientific, 10003D) were added and the incubation continued for 2 additional h. Beads were extensively washed at $\mathrm{RT}^{66}$. DNA was eluted twice with $57 \mu$ of elution buffer (1\% (w/v) SDS in TE pH 8.0 with $250 \mathrm{mM} \mathrm{NaCl}$ ) at $65^{\circ} \mathrm{C}, 15 \mathrm{~min}$ each time. Eluted DNA was incubated with $20 \mu \mathrm{g}$ of RNase A for $30 \mathrm{~min}$ at $37^{\circ} \mathrm{C}$ and then with $20 \mu \mathrm{g}$ of Proteinase $\mathrm{K}$ for $1 \mathrm{~h}$ at $55^{\circ} \mathrm{C}$. Input DNA was also diluted in $114 \mu \mathrm{l}$ of elution buffer and treated as ChIP samples. Crosslinks were reversed at $65^{\circ} \mathrm{C}$ overnight and DNA was purified using the QIAGEN MinElute PCR Purification Kit (QIAGEN, 28006) according to the manufacturer's instructions (Qiagen). DNA concentration was measured with a Qubit Fluorometer and fragment size was determined with a Fragment analyzer. For ChIP-seq, libraries were prepared using the NEBNext Ultra II DNA Library Prep Kit (New England BioLabs, E7645S) as per manufacturer's instructions. Libraries were amplified for 5-7 total PCR cycles, purified with AMPure XP beads and sequenced on a Next-Seq 500 Sequencer (Illumina) at the DanStem Genomics Platform (University of Copenhagen, Copenhagen, Denmark). For ChIP-qPCR, immunoprecipitated DNA and input were quantified by real-time qPCR as described below. The following primers and probes (Universal Probe Library, UPL, Roche) were used: for Msgn1 PBX/HOX-1-binding site, Fw, GTGCAGAAATATCCCCTGCT; Rv, TGTCTTCTGGTGCTGTTTGC; Probe, 95; for Msgn1 LEF1-binding site, Fw, GCATCTTCCAGCTAAAATGTCTTT; Rv, GCAGCCTTAATTGGTTTAGTTACAA; Probe, 75. The measures were normalised to the input. All reactions were performed in duplicates.

Real-time quantitative PCR. Total RNA was extracted from $0.5-1 \times 10^{6}$ cells using the RNeasy Mini Kit according to the manufacturer's instructions. Firststrand synthesis was performed on $1 \mu \mathrm{g}$ of total RNA using random hexamers (Thermo Fisher Scientific, N8080127) and SuperScript III reverse transcriptase (Thermo Fisher Scientific, 18080-044) according to the manufacturer's instructions. A mix of concentrated cDNA from all samples was used to generate standard curves. Amplification was detected using the Universal Probe Library system on a LightCycler480 Real-Time PCR System (Roche). The measures were normalised to Tbp and Sdha reference genes. All reactions were performed in duplicates. See Supplementary Table 5 for a list of primers and probes used.

scRNA-seq data analysis. $\mathrm{R} 1$ and $\mathrm{R} 2$ fastq files were generated using bcl2fastq (v2.19.1), and the pooling and well information were extracted from the sequence using umis (v1.0.3) [https://github.com/vals/umis] into a unique fastq file. The reads were then filtered based on the pooling barcodes with 1 mismatch allowed. The poly-Ts at the end of the reads were trimmed using Cutadapt (v1.18) ${ }^{67}$. The reads were mapped to the mouse genome (GRCm38/mm10 together with ERCC92) using HISAT2 (v2.1.0), the alignments were processed with Samtools $(\mathrm{v} 1.7)^{68}$, and the reads were counted with featureCounts (Subread (v1.5.3) ${ }^{69}$ using Ensembl v93, and the umis using UMI_tools (v1.0.0) ${ }^{70}$. Expression data were analysed using Seurat (v3.1) ${ }^{71,72}$. Data filtering, normalisation and scaling were performed using the standard pre-processing workflow ${ }^{71}$. Integration of different datasets was performed as described in Stuart et al. ${ }^{72}$. Spiked-in EpiSCs were used as a reference to correct the batch-effect between integrated datasets. Marker genes of each cell cluster were outputted for GO-term analysis to define the cell type. The Monocle package (v2.16.0 $)^{73}$ was used to perform pseudotime analyses. Single-cell trajectories were constructed in a semi-supervised manner, by defining NMPs as cells co-expressing moderate levels of T-Bra/Sox2, MPCs as co-expressing high levels of T-Bra/Tbx6 and PSM as cells co-expressing high levels of Tbx6/Meox1.
RNA-seq data analysis. Fastq files were aligned to the GRCm $38 / \mathrm{mm} 10$ genome using STAR (v2.5.3a) ${ }^{74}$. Transcript expression levels were estimated with the-quantMode GeneCounts option and GRCm38p5.vM15 annotations. FastQC (v0.11.7) (http://www.bioinformatics.babraham.ac.uk/projects/fastqc) was used for QC metrics, and MultiQC (v1.7) ${ }^{75}$ for reporting. Data analysis was then performed with R/Biocondutcor (v4.0.5) (https://www.R-project.org) ${ }^{76}$. Differentially expressed genes were identified using DESeq2 (v1.24.0) ${ }^{77}$, which applies negative binomial generalised linear model fitting and Wald statistics on the count data. The results were filtered for Benjamini-Hochberg adjusted $P \leq 0.05$ and fold-change $\geq 1.5$, as indicated in the figure legends.

GO-term analysis. Biological pathway enrichments were determined using Panther $(\mathrm{v} 15.0)^{78}$. Statistical significance was assessed using Binomial test with Bonferroni correction for multiple testing $(P \leq 0.05)$. Only the top 12 terms ordered by Fold enrichment were considered and ranked by $P$-value.

ATAC-seq data analysis. Reads were trimmed with Cutadapt (v1.18) ${ }^{79}$ (Cutadapt -a CTGTCTCTTATA -A CTGTCTCTTATA -q 20 -m 5), mapped to the GRCm38/ $\mathrm{mm} 10$ mouse reference genome with Bowtie2 (v2.3.4.1) ${ }^{80}$. Mitochondrial reads and reads overlapping blacklisted regions (i.e. regions that are considered artefacts of ATAC-seq and other chromatin assays in mm10, https://github.com/MayurDivate/ GUAVA/blob/master/lib/blacklists/JDB_blacklist.mml0.bed and http://mitra.stanford. edu/kundaje/akundaje/release/blacklists/mm10-mouse) were removed using bedtools (v2.27.1 $)^{81}$, intersect and only uniquely mapped reads were kept using Samtools $(\mathrm{v} 1.9)^{82}$. Bam files were sorted, deduplicated with Picard (v2.9.1) (http://broadinstitute. github.io/picard/) and indexed. Bigwig files were generated with bamCoverage (v3.2.0) from deepTools ${ }^{83}$ and the RPGC option. MACS (v2.1.1.20160309) was used to call peaks 84 .

ChIP-seq data analysis. Reads were mapped to the GRCm $38 / \mathrm{mm} 10$ reference genome with Bowtie2 (v2.3.4.1) ${ }^{80}$ and only uniquely mapped reads were kept using Samtools (v1.9) ${ }^{82}$. Bam files were sorted, deduplicated and indexed. Bigwig files were generated with bamCoverage (v3.2.0) from deepTools ${ }^{83}$ and the RPGC option. The effective genome size was taken from the deepTools documentation. MACS (v2.1.1.20160309) was used to call peaks ${ }^{84}$. Heatmaps and peak profile were generated from the bigwig files with normalised counts to RPKM using deepTools $(\mathrm{v} 3.2 .0)^{82}$. The relative distance between peaks was calculated using the closest function in bedtools $(\mathrm{v} 2.27 .1)^{81}$.

De novo motif enrichment analysis. Homer (v4.1) ${ }^{85}$ de novo motif enrichment analysis was performed on bed files generated by MACS (v2.1.1.20160309) ${ }^{84}$ peak caller on the GRCm $38 / \mathrm{mm} 10$ reference genome with default settings.

Venn diagrams. Proportional Venn diagrams to show unique and intersecting elements of gene lists were generated using the web tool available at https:// www.biovenn.nl.

Statistics and reproducibility. The sample size was estimated based on previous preliminary experiments. No statistical method was used to pre-determine sample size. Experiments and quantifications were not done in a blinded fashion. Unless otherwise noted, each immunofluorescence staining was repeated at least three times independently with similar results. Each western blot and EMSA were performed at least twice independently with similar results. Data are presented in box plots as median \pm interquartile range as indicated in the figure legends, when $n \geq 10$. Data are presented in histograms when $n<10$, and individual data points are plotted. Information about $P$-values and statistical tests used in this article can be found in the figure legends. Statistics were derived using GraphPad Prism (v8.4.3) (GraphPad Software, San Diego, CA, USA). The raw data underlying all reported averages in graphs and charts, and the uncropped scans of western blots and EMSAs are provided in the Source data file.

Reporting summary. Further information on experimental design is available in the Nature Research Reporting Summary linked to this paper.

\section{Data availability}

All data generated during this study are included in this article and its Supplementary Information files. scRNA-seq, bulk RNA-seq, ATAC-seq and ChIP-seq data that support the findings of this study have been deposited in the ArrayExpress database at EMBL-EBI (www.ebi.ac.uk/arrayexpress) under the following accession numbers: scRNA-seq of embryonic tailbuds, E-MTAB-9785; scRNA-seq of in vitro cells, E-MTAB-9774; bulk RNA-seq, E-MTAB-9773; ATAC-seq, E-MTAB-9776; and ChIP-seq, E-MTAB-9775. Source data are provided with this paper.

Received: 25 February 2021; Accepted: 4 August 2021; Published online: 26 August 2021 


\section{References}

1. Aires, R., Dias, A. \& Mallo, M. Deconstructing the molecular mechanisms shaping the vertebrate body plan. Curr. Opin. Cell Biol. 55, 81-86 (2018).

2. Stern, C. D. et al. Head-tail patterning of the vertebrate embryo: one, two or many unresolved problems? Int. J. Dev. Biol. 50, 3-15 (2006).

3. Wilson, V., Olivera-Martinez, I. \& Storey, K. G. Stem cells, signals and vertebrate body axis extension. Development 136, 1591-1604 (2009).

4. Cambray, N. \& Wilson, V. Two distinct sources for a population of maturing axial progenitors. Development 134, 2829-2840 (2007).

5. Henrique, D., Abranches, E., Verrier, L. \& Storey, K. G. Neuromesodermal progenitors and the making of the spinal cord. Development 142, 2864-2875 (2015).

6. Steventon, B. \& Martinez Arias, A. Evo-engineering and the cellular and molecular origins of the vertebrate spinal cord. Dev. Biol. 432, 3-13 (2017).

7. Cambray, N. \& Wilson, V. Axial progenitors with extensive potency are localised to the mouse chordoneural hinge. Development 129, 4855-4866 (2002).

8. Tzouanacou, E., Wegener, A., Wymeersch, F. J., Wilson, V. \& Nicolas, J. F. Redefining the progression of lineage segregations during mammalian embryogenesis by clonal analysis. Dev. Cell 17, 365-376 (2009).

9. Amin, S. et al. Cdx and T brachyury co-activate growth signaling in the embryonic axial progenitor niche. Cell Rep. 17, 3165-3177 (2016).

10. Chalamalasetty, R. B. et al. Mesogenin 1 is a master regulator of paraxial presomitic mesoderm differentiation. Development 141, 4285-4297 (2014).

11. Denans, N., Iimura, T. \& Pourquie, O. Hox genes control vertebrate body elongation by collinear Wnt repression. Elife https://doi.org/10.7554/ eLife.04379 (2015).

12. Garriock, R. J. et al. Lineage tracing of neuromesodermal progenitors reveals novel Wnt-dependent roles in trunk progenitor cell maintenance and differentiation. Development 142, 1628-1638 (2015).

13. Koch, F. et al. Antagonistic activities of Sox 2 and brachyury control the fate choice of neuro-mesodermal progenitors. Dev. Cell 42, 514-526 e517 (2017).

14. Nowotschin, S., Ferrer-Vaquer, A., Concepcion, D., Papaioannou, V. E. \& Hadjantonakis, A. K. Interaction of Wnt3a, Msgn1 and Tbx6 in neural versus paraxial mesoderm lineage commitment and paraxial mesoderm differentiation in the mouse embryo. Dev. Biol. 367, 1-14 (2012).

15. Wittler, L. et al. Expression of Msgn1 in the presomitic mesoderm is controlled by synergism of WNT signalling and Tbx6. EMBO Rep. 8, 784-789 (2007).

16. Young, T. \& Deschamps, J. Hox, Cdx, and anteroposterior patterning in the mouse embryo. Curr. Top. Dev. Biol. 88, 235-255 (2009).

17. Diaz-Cuadros, M. et al. In vitro characterization of the human segmentation clock. Nature 580, 113-118 (2020).

18. Gouti, M. et al. A gene regulatory network balances neural and mesoderm specification during vertebrate trunk development. Dev. Cell 41, 243-261.e247 (2017).

19. Iimura, T. \& Pourquie, O. Collinear activation of Hoxb genes during gastrulation is linked to mesoderm cell ingression. Nature 442, 568-571 (2006).

20. Deschamps, J. \& Duboule, D. Embryonic timing, axial stem cells, chromatin dynamics, and the Hox clock. Genes Dev. 31, 1406-1416 (2017).

21. Young, T. et al. Cdx and Hox genes differentially regulate posterior axial growth in mammalian embryos. Dev. Cell 17, 516-526 (2009).

22. Selleri, L., Zappavigna, V. \& Ferretti, E. 'Building a perfect body': control of vertebrate organogenesis by PBX-dependent regulatory networks. Genes Dev. 33, 258-275 (2019).

23. Rezsohazy, R., Saurin, A. J., Maurel-Zaffran, C. \& Graba, Y. Cellular and molecular insights into Hox protein action. Development 142, 1212-1227 (2015).

24. Capellini, T. D. et al. Pbx1/Pbx2 requirement for distal limb patterning is mediated by the hierarchical control of Hox gene spatial distribution and Shh expression. Development 133, 2263-2273 (2006).

25. Capellini, T. D. et al. Pbx1/Pbx2 govern axial skeletal development by controlling Polycomb and Hox in mesoderm and Pax1/Pax9 in sclerotome. Dev. Biol. 321, 500-514 (2008).

26. Sonnet, W., Rezsohazy, R. \& Donnay, I. Characterization of TALE genes expression during the first lineage segregation in mammalian embryos. Dev. Dyn. 241, 1827-1839 (2012).

27. Mariani, L. et al. Identification of the enhancer signature priming lineage choice at gastrulation. In preparation.

28. Wymeersch, F. J. et al. Transcriptionally dynamic progenitor populations organised around a stable niche drive axial patterning. Development https:// doi.org/10.1242/dev.168161 (2019).

29. Berkes, C. A. et al. Pbx marks genes for activation by MyoD indicating a role for a homeodomain protein in establishing myogenic potential. Mol. Cell 14, 465-477 (2004).

30. Grebbin, B. M. \& Schulte, D. PBX1 as pioneer factor: a case still open. Front. Cell Dev. Biol. 5, 9 (2017).

31. Magnani, L., Ballantyne, E. B., Zhang, X. \& Lupien, M. PBX1 genomic pioneer function drives ERalpha signaling underlying progression in breast cancer. PLoS Genet. 7, e1002368 (2011).
32. Penalosa-Ruiz, G., Bright, A. R., Mulder, K. W. \& Veenstra, G. J. C. The interplay of chromatin and transcription factors during cell fate transitions in development and reprogramming. Biochim. Biophys. Acta Gene Regul. Mech. 1862, 194407 (2019).

33. Penkov, D. et al. Analysis of the DNA-binding profile and function of TALE homeoproteins reveals their specialization and specific interactions with Hox genes/proteins. Cell Rep. 3, 1321-1333 (2013).

34. Mosimann, C., Hausmann, G. \& Basler, K. Beta-catenin hits chromatin: regulation of Wnt target gene activation. Nat. Rev. Mol. Cell Biol. 10, 276-286 (2009).

35. Galceran, J., Farinas, I., Depew, M. J., Clevers, H. \& Grosschedl, R. Wnt3a-/like phenotype and limb deficiency in Lef1(-/-)Tcf1(-/-) mice. Genes Dev. 13, 709-717 (1999).

36. Merabet, S. et al. Insights into Hox protein function from a large scale combinatorial analysis of protein domains. PLoS Genet. 7, e1002302 (2011)

37. Mann, R. S., Lelli, K. M. \& Joshi, R. Hox specificity unique roles for cofactors and collaborators. Curr. Top. Dev. Biol. 88, 63-101 (2009).

38. Tosic, J. et al. Eomes and Brachyury control pluripotency exit and germ-layer segregation by changing the chromatin state. Nat. Cell Biol. 21, 1518-1531 (2019).

39. Mallo, M. \& Alonso, C. R. The regulation of Hox gene expression during animal development. Development 140, 3951-3963 (2013).

40. Zeitlinger, J. Seven myths of how transcription factors read the cis-regulatory code. Curr. Opin. Syst. Biol. 23, 22-31 (2020).

41. Choe, S. K., Lu, P., Nakamura, M., Lee, J. \& Sagerstrom, C. G. Meis cofactors control HDAC and CBP accessibility at Hox-regulated promoters during zebrafish embryogenesis. Dev. Cell 17, 561-567 (2009)

42. Dell'Orso, S. et al. The histone variant macroH2A1.2 is necessary for the activation of muscle enhancers and recruitment of the transcription factor Pbx1. Cell Rep. 14, 1156-1168 (2016).

43. Mirny, L. A. Nucleosome-mediated cooperativity between transcription factors. Proc. Natl Acad. Sci. USA 107, 22534-22539 (2010).

44. Porcelli, D., Fischer, B., Russell, S. \& White, R. Chromatin accessibility plays a key role in selective targeting of Hox proteins. Genome Biol. 20, 115 (2019).

45. Ladam, F. et al. TALE factors use two distinct functional modes to control an essential zebrafish gene expression program. Elife https://doi.org/10.7554/ eLife.36144 (2018)

46. Steventon, B. et al. Species-specific contribution of volumetric growth and tissue convergence to posterior body elongation in vertebrates. Development 143, 1732-1741 (2016).

47. Takemoto, T. et al. Tbx6-dependent Sox2 regulation determines neural or mesodermal fate in axial stem cells. Nature 470, 394-398 (2011).

48. Aulehla, A. \& Pourquie, O. Signaling gradients during paraxial mesoderm development. Cold Spring Harb. Perspect. Biol. 2, a000869 (2010).

49. Phan, Q. M. et al. Lef1 expression in fibroblasts maintains developmental potential in adult skin to regenerate wounds. Elife https://doi.org/10.7554/ eLife.60066 (2020).

50. Niemann, C., Owens, D. M., Schettina, P. \& Watt, F. M. Dual role of inactivating Lef1 mutations in epidermis: tumor promotion and specification of tumor type. Cancer Res. 67, 2916-2921 (2007).

51. Morgan, R. et al. Antagonism of HOX/PBX dimer formation blocks the in vivo proliferation of melanoma. Cancer Res. 67, 5806-5813 (2007).

52. Ying, Q. L. \& Smith, A. G. Defined conditions for neural commitment and differentiation. Methods Enzymol. 365, 327-341 (2003).

53. Koss, M. et al. Congenital asplenia in mice and humans with mutations in a $\mathrm{Pbx} / \mathrm{Nkx} 2-5 / \mathrm{p} 15$ module. Dev. Cell 22, 913-926 (2012).

54. Selleri, L. et al. The TALE homeodomain protein Pbx2 is not essential for development and long-term survival. Mol. Cell Biol. 24, 5324-5331 (2004).

55. Hayashi, S., Lewis, P., Pevny, L. \& McMahon, A. P. Efficient gene modulation in mouse epiblast using a Sox2Cre transgenic mouse strain. Mech. Dev. 119, S97-S101 (2002).

56. Guo, G. et al. Klf4 reverts developmentally programmed restriction of ground state pluripotency. Development 136, 1063-1069 (2009).

57. Ran, F. A. et al. Double nicking by RNA-guided CRISPR Cas9 for enhanced genome editing specificity. Cell 154, 1380-1389 (2013).

58. Kleinstiver, B. P. et al. High-fidelity CRISPR-Cas 9 nucleases with no detectable genome-wide off-target effects. Nature 529, 490-495 (2016)

59. Loh, K. M. et al. Mapping the pairwise choices leading from pluripotency to human bone, heart, and other mesoderm cell types. Cell 166, 451-467 (2016).

60. Yoon, J. K. \& Wold, B. The bHLH regulator pMesogenin1 is required for maturation and segmentation of paraxial mesoderm. Genes Dev. 14, 3204-3214 (2000).

61. Parr, B. A., Shea, M. J., Vassileva, G. \& McMahon, A. P. Mouse Wnt genes exhibit discrete domains of expression in the early embryonic CNS and limb buds. Development 119, 247-261 (1993).

62. Ferretti, E. et al. Segmental expression of Hoxb2 in r4 requires two separate sites that integrate cooperative interactions between Prep1, Pbx and Hox proteins. Development 127, 155-166 (2000). 
63. Jaitin, D. A. et al. Massively parallel single-cell RNA-seq for marker-free decomposition of tissues into cell types. Science 343, 776-779 (2014).

64. Buenrostro, J. D., Giresi, P. G., Zaba, L. C., Chang, H. Y. \& Greenleaf, W. J. Transposition of native chromatin for fast and sensitive epigenomic profiling of open chromatin, DNA-binding proteins and nucleosome position. Nat. Methods 10, 1213-1218 (2013).

65. Corces, M. R. et al. An improved ATAC-seq protocol reduces background and enables interrogation of frozen tissues. Nat. Methods 14, 959-962 (2017).

66. Kolasinska-Zwierz, P. et al. Differential chromatin marking of introns and expressed exons by H3K36me3. Nat. Genet. 41, 376-381 (2009).

67. Martin, M. Cutadapt removes adapter sequences from high-throughput sequencing reads. EMBnet. J. 2011, 10-12 (2011).

68. Kim, D., Langmead, B. \& Salzberg, S. L. HISAT: a fast spliced aligner with low memory requirements. Nat. Methods 12, 357-360 (2015).

69. Liao, Y., Smyth, G. K. \& Shi, W. featureCounts: an efficient general purpose program for assigning sequence reads to genomic features. Bioinformatics $\mathbf{3 0}$, 923-930 (2014).

70. Smith, T., Heger, A. \& Sudbery, I. UMI-tools: modeling sequencing errors in Unique Molecular Identifiers to improve quantification accuracy. Genome Res. 27, 491-499 (2017).

71. Butler, A., Hoffman, P., Smibert, P., Papalexi, E. \& Satija, R. Integrating singlecell transcriptomic data across different conditions, technologies, and species. Nat. Biotechnol. 36, 411-420 (2018).

72. Stuart, T. et al. Comprehensive integration of single-cell data. Cell 177, 1888-1902.e1821 (2019).

73. Trapnell, C. et al. The dynamics and regulators of cell fate decisions are revealed by pseudotemporal ordering of single cells. Nat. Biotechnol. 32, 381-386 (2014).

74. Dobin, A. et al. STAR: ultrafast universal RNA-seq aligner. Bioinformatics 29, 15-21 (2013)

75. Ewels, P., Magnusson, M., Lundin, S. \& Kaller, M. MultiQC: summarize analysis results for multiple tools and samples in a single report. Bioinformatics 32, 3047-3048 (2016).

76. Gentleman, R. C. et al. Bioconductor: open software development for computational biology and bioinformatics. Genome Biol. 5, R80 (2004).

77. Love, M. I., Huber, W. \& Anders, S. Moderated estimation of fold change and dispersion for RNA-seq data with DESeq2. Genome Biol. 15, 550 (2014).

78. Thomas, P. D. et al. PANTHER: a library of protein families and subfamilies indexed by function. Genome Res. 13, 2129-2141 (2003).

79. Martin, D. P., Lemey, P. \& Posada, D. Analysing recombination in nucleotide sequences. Mol. Ecol. Resour. 11, 943-955 (2011).

80. Langmead, B. \& Salzberg, S. L. Fast gapped-read alignment with Bowtie 2. Nat. Methods 9, 357-359 (2012).

81. Quinlan, A. R. \& Hall, I. M. BEDTools: a flexible suite of utilities for comparing genomic features. Bioinformatics 26, 841-842 (2010).

82. $\mathrm{Li}, \mathrm{H}$. et al. The sequence alignment/map format and SAMtools. Bioinformatics 25, 2078-2079 (2009)

83. Ramirez, F. et al. deepTools2: a next generation web server for deepsequencing data analysis. Nucleic Acids Res. 44, W160-W165 (2016).

84. Zhang, Y. et al. Model-based analysis of ChIP-Seq (MACS). Genome Biol. 9, R137 (2008).

85. Heinz, S. et al. Simple combinations of lineage-determining transcription factors prime cis-regulatory elements required for macrophage and B cell identities. Mol. Cell 38, 576-589 (2010).

\section{Acknowledgements}

We thank the staff of the DanStem Core Facilities: H.M. Neil, M. Michaut, G. De la Cruz, P. van Dieken, J.M. Bulkescher, A. Shrestha and A. Meligkova. We are grateful to M.M. Rothová for sharing the wild-type 129 EpiSC line, L. Selleri for providing the $P b x$ mutant alleles and the Core Facility for Transgenic Mice at the University of Copenhagen, especially J. Martin Gonzalez for derivation of mouse and EpiSC lines. We thank J.M. Brickman, W. Hamilton and J. Zylicz for critical reading of the manuscript and extensive discussion. This study is supported by the Novo Nordisk Foundation grant number NNF17CC0027852, and the Danish Council for Independent Research project grant 4183-00516A. Work supported by Marie Curie Reintegration Fellowship (H2020-MSCA-IF-2014_RI) to E.F.; and Lundbeckfonden PhD Fellowship (grant number R180-2014-2980) to X.G.

\section{Author contributions}

L.M., X.G., N.A.M., A.M.D. and E.F. conceived, performed and analysed the experiments. X.G., N.A.M., L.M. and Q.W. performed the bioinformatics data analyses. X.G. and L.M. designed the CRISPR/Cas9 strategies. A.M.D. performed the lentiviral experiments. S.D.Ç. generated the $P b x 1^{f l / f l} ; \mathrm{Pbx}^{-/}$;Crepos EpiSC cell lines. L.M., X.G., N.A.M. and E.F. prepared the figures, wrote and edited the manuscript.

\section{Competing interests}

The authors declare no competing interests.

\section{Additional information}

Supplementary information The online version contains supplementary material available at https://doi.org/10.1038/s41467-021-25370-4.

Correspondence and requests for materials should be addressed to E.F.

Peer review information Nature Communications thanks Hugo J Parker, Valerie Wilson and other, anonymous, reviewers for their contributions to the peer review of this work. Peer review reports are available.

Reprints and permission information is available at http://www.nature.com/reprints

Publisher's note Springer Nature remains neutral with regard to jurisdictional claims in published maps and institutional affiliations.

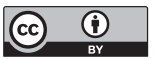

Open Access This article is licensed under a Creative Commons Attribution 4.0 International License, which permits use, sharing, adaptation, distribution and reproduction in any medium or format, as long as you give appropriate credit to the original author(s) and the source, provide a link to the Creative Commons license, and indicate if changes were made. The images or other third party material in this article are included in the article's Creative Commons license, unless indicated otherwise in a credit line to the material. If material is not included in the article's Creative Commons license and your intended use is not permitted by statutory regulation or exceeds the permitted use, you will need to obtain permission directly from the copyright holder. To view a copy of this license, visit http://creativecommons.org/ licenses/by/4.0/

(c) The Author(s) 2021 